\title{
Probing the first galaxies with the Square Kilometer Array
}

\author{
M. G. Santos ${ }^{1}$, M. B. Silva ${ }^{1}$, J. R. Pritchard ${ }^{2}{ }^{\star}$, R. Cen $^{3}$, and A. Cooray ${ }^{4}$ \\ ${ }^{1}$ CENTRA, Departamento de Física, Instituto Superior Técnico, 1049-001 Lisboa, Portugal \\ e-mail: mgrsantos@ist.utl.pt \\ 2 Harvard-Smithsonian Center for Astrophysics, Cambridge, MA 02138, USA \\ 3 Department of Astrophysical Sciences, Princeton University, Princeton, NJ 08544, USA \\ ${ }^{4}$ Center for Cosmology, Department of Physics and Astronomy, University of California, Irvine, CA 92697, USA
}

Received 5 September 2010 / Accepted 26 December 2010

\begin{abstract}
Observations of anisotropies in the brightness temperature of the $21 \mathrm{~cm}$ line of neutral hydrogen from the period before reionization would shed light on the dawn of the first stars and galaxies. In this paper, we use large-scale semi-numerical simulations to analyse the imprint on the $21 \mathrm{~cm}$ signal of spatial fluctuations in the Lyman- $\alpha$ flux arising from the clustering of the first galaxies. We show that an experiment such as the Square Kilometer Array (SKA) can probe this signal at the onset of reionization, giving us important information about the UV emission spectra of the first stars and characterizing their host galaxies. SKA-pathfinders with $\sim 10 \%$ of the full collecting area should be capable of making a statistical detection of the $21 \mathrm{~cm}$ power spectrum at redshifts $z \lesssim 20$ (corresponding to frequencies $v \gtrsim 67 \mathrm{MHz}$ ). We then show that the SKA should be able to measure the three dimensional power spectrum as a function of the angle with the line of sight and discuss the use of the redshift space distortions as a way to separate out the different components of the $21 \mathrm{~cm}$ power spectrum. We demonstrate that, at least on large scales where the Ly $\alpha$ fluctuations are linear, they can be used as a model independent way to extract the power spectra due to these Ly $\alpha$ fluctuations.
\end{abstract}

Key words. cosmology: miscellaneous - large-scale structure of Universe - galaxies: high-redshift - intergalactic medium

\section{Introduction}

The period of formation of the very first stars is one of the least understood epochs in the history of the Universe. The $21 \mathrm{~cm}$ spin-flip transition of neutral hydrogen at high redshifts has the potential to open a new observational window to study this early period, where the most distant, first galaxies reside, and even beyond that. Moreover, $21 \mathrm{~cm}$ observations may provide the only means to detect signatures of the first galaxies at $z>15$ in the foreseeable future. While the James Webb Space Telescope (JWST ${ }^{1}$ ) will be able to detect galaxies at $z \leq 10$ (e.g., Stiavelli et al. 2004), it will not image all of the sources responsible for the reionization of the Universe. If current estimates in the literature are correct, even deep fields with JWST will only detect $\sim 30 \%$ of the sources responsible for reionization or maintaining reionization at $z \sim 12$ (Salvaterra et al. 2010). At $z \sim 20$, direct emission from stars will be below the threshold of JWST observations and new techniques will be required to probe the onset of reionization. One possibility is Lyman- $\alpha$ emitter surveys over the next decade, but even these surveys will only be able to detect the brightest galaxies for selected redshift ranges at $z \leq 10$ (e.g., Ouchi et al. 2008; Nilsson et al. 2007; Cuby et al. 2007; Stark et al. 2007; Willis et al. 2008; McMahon et al. 2008; Hibon et al. 2010), but not the fainter, first galaxies at redshift $z \geq 20$. The next generation of large ground based telescopes, like the European Extremely Large Telescope (E-ELT $\left.{ }^{2}\right)$, Thirty Meter Telescope $\left(\mathrm{TMT}^{3}\right)$, and Giant Magellan Telescope $\left(\mathrm{GMT}^{4}\right)$, will

\footnotetext{
^ Hubble Fellow.

1 http://www. jwst. nasa.gov/

2 http://www.eelt.org

3 http://www.tmt.org/

${ }^{4}$ http://www.gmto.org/
}

only be capable of detecting at most the very brightest galaxies at redshifts $z \approx 20$. Most of the star formation however will be in fainter galaxies making it impossible for these telescopes alone to survey the full population. In combination with $21 \mathrm{~cm}$ observations, which are sensitive to the integrated Ly $\alpha$ emission of the galaxies, it should be possible to form a complete census of the first sources.

With a wide frequency coverage, observations of the $21 \mathrm{~cm}$ signal will provide a 3-dimensional tomographic view of the inter-galactic medium (IGM), before and during the cosmological reionization (for a review see Furlanetto et al. 2006). As soon as the first galaxies appear, at a redshift of $z \sim 20-30$ in the standard cold dark matter cosmological model (e.g., Komatsu et al. 2011), photons emitted at frequencies between the Ly $\alpha$ and Lyman limit quickly couple the spin temperature of the hyperfine level population to the IGM gas temperature through the Wouthuysen-Field effect (Wouthuysen 1952; Field 1959). Provided the IGM has been cooling adiabatically (X-ray heating is still negligible during this early stage of galaxy formation), the $21 \mathrm{~cm}$ signal will be strong and observed in absorption against the cosmic microwave background (CMB). Because the Ly $\alpha$ coupling depends on the local Ly $\alpha$ radiation, which is correlated with galaxies, the $21 \mathrm{~cm}$ signal fluctuations will trace the location, mass, emission spectra, luminosity and redshift evolution of high redshift galaxies.

Since the first galaxies represent rare high- $\sigma$ peaks in the cosmic density field (e.g., Trac \& Cen 2007), their spatial distribution have large fluctuations and we expect the Ly $\alpha$ contribution to the overall $21 \mathrm{~cm}$ signal to be appreciable, considerably improving the prospects for detection of the Ly $\alpha$ fluctuations through $21 \mathrm{~cm}$ observations. Although the high redshiftend of the reionization process will be inaccessible to the first 
generation experiments, such as the LOw Frequency ARray $\left(\mathrm{LOFAR}^{5}\right)$ and the Murchison Widefield Array $\left(\mathrm{MWA}^{6}\right)$, the second generation experiments such as the Square Kilometre Array $\left(\mathrm{SKA}^{7}\right)$ should have enough collecting area to statistically probe the signal.

On the theoretical front, analytical models have been useful in predicting the probable evolution of the high redshift signal (Barkana \& Loeb 2005b; Pritchard \& Furlanetto 2007; Pritchard \& Loeb 2008), but rely on linear approximations and require further comparison with simulations. Numerical simulations, on the other hand, can provide a self-consistent treatment of the Ly $\alpha$ radiative transfer, taking into account effects such as the scattering in the Ly $\alpha$ line wings (Semelin et al. 2007; Baek et al. 2009), although they are typically slow to run and are limited to small volumes ( $\leq 100 \mathrm{Mpc} / \mathrm{h})$. Recent developments using seminumerical algorithms have allowed the rapid generation of the high redshift $21 \mathrm{~cm}$ signal during the pre-reionization epoch (Santos et al. 2008, 2010; Mesinger et al. 2010) in large boxes, while maintaining the 3 -d structure of the signal on large scales as seen in the full numerical simulations.

In this paper we will generate fast simulations as described in Santos et al. (2010) to analyze the dependence of the overall $21 \mathrm{~cm}$ signal on several parameters related to the first galaxies in the Universe and consider the ability of an experiment like SKA to constrain these parameters. The layout of this paper is as follows. We start in Section 2 by describing the $21 \mathrm{~cm}$ signal and the free parameters of the model that affect the Ly $\alpha$ fluctuations. In Sect. 3 we present the experimental setup used for SKA, calculating the expected error on the 3-d power spectrum. In Sect. 4 we study the possibility of constraining the signal in a model independent way, using the redshift-space distortions and the corresponding measurements on the 3 -d power spectrum as a function of the angle with respect to the line of sight. We end with our conclusions and a discussion of the prospects for SKA in Sect. 5.

Throughout this paper where cosmological parameters are required we use the standard set of values $\Omega_{\mathrm{m}}=0.28, \Omega_{\Lambda}=$ $0.72, \Omega_{\mathrm{b}}=0.046, H=100 h \mathrm{~km} \mathrm{~s}^{-1} \mathrm{Mpc}^{-1}$ (with $h=0.70$ ), $n_{\mathrm{S}}=0.96$, and $\sigma_{8}=0.82$, consistent with the latest measurements (Komatsu et al. 2011).

\section{The $21 \mathrm{~cm}$ signal: Ly $\alpha$ fluctuations}

The $21 \mathrm{~cm}$ brightness temperature corresponds to the change in the intensity of the $\mathrm{CMB}$ radiation due to absorption or emission when it travels through a patch of neutral hydrogen. It is given, at an observed frequency $v$ in the direction $\hat{\mathbf{n}}$, by (see e.g. Santos et al. 2008)

$$
\begin{aligned}
\delta T_{\mathrm{b}}(v) \approx & 23 x_{H I}(1+\delta)\left(1-\frac{T_{\gamma}}{T_{\mathrm{S}}}\right)\left(\frac{h}{0.7}\right)^{-1}\left(\frac{\Omega_{\mathrm{b}} h^{2}}{0.02}\right) \\
& \times\left[\left(\frac{0.15}{\Omega_{\mathrm{m}} h^{2}}\right)\left(\frac{1+z}{10}\right)\right]^{1 / 2}\left(\frac{1}{1+1 / H \mathrm{~d} v_{r} / \mathrm{d} r}\right) \mathrm{mK},
\end{aligned}
$$

where $x_{\mathrm{HI}}$ is the fraction of neutral hydrogen (mass weighted), $\mathrm{d} v_{r} / \mathrm{d} r$ is the comoving gradient of the line of sight component of the comoving velocity and we use $\delta_{a}$ for the fractional value of the quantity $a\left(\delta_{a} \equiv \frac{a-\langle a\rangle}{\langle a\rangle}\right)$ with $\delta$ for the fluctuation in the matter density.

\footnotetext{
5 http://www.lofar.org

6 http://www. mwatelescope.org

7 http://www. skatelescope.org
}

Table 1. Simulations used in the analysis.

\begin{tabular}{cccc}
\hline \hline & Size & $\begin{array}{c}\text { Resolution } \\
\text { (halos) }\end{array}$ & $\begin{array}{c}\text { Resolution } \\
(\text { Ly } \alpha)\end{array}$ \\
\hline S1 & $1000 \mathrm{Mpc}$ & $0.56 \mathrm{Mpc}$ & $1.667 \mathrm{Mpc}$ \\
S2 & $143 \mathrm{Mpc}$ & $0.09 \mathrm{Mpc}$ & $0.186 \mathrm{Mpc}$ \\
S3 $(N$-body) & $143 \mathrm{Mpc}$ & - & $0.186 \mathrm{Mpc}$ \\
\hline
\end{tabular}

Notes. Middle column shows the resolution used to resolve halos in the semi-numerical code, while the last column shows the resolution used in the $\operatorname{Ly} \alpha$ calculation.

The spin temperature $\left(T_{\mathrm{S}}\right)$ is coupled to the hydrogen gas temperature $\left(T_{K}\right)$ through the spin-flip transition, which can be excited by collisions or by the absorption of Ly $\alpha$ photons (Wouthuysen-Field effect) and we can write:

$1-\frac{T_{\gamma}}{T_{\mathrm{S}}}=\frac{x_{\mathrm{tot}}}{1+x_{\mathrm{tot}}}\left(1-\frac{T_{\gamma}}{T_{K}}\right)$,

where $x_{\mathrm{tot}}=x_{\alpha}+x_{\mathrm{c}}$ is the sum of the radiative and collisional coupling parameters and we are already assuming that the color temperature of the $\operatorname{Ly} \alpha$ radiation field at the $\operatorname{Ly} \alpha$ frequency is equal to $T_{K}$. Note that although we talk here about Ly $\alpha$ radiation, in effect we consider the contribution from all the photons up to the Lyman limit frequency, since photons redshifting into Lyman series resonances can produce $\operatorname{Ly} \alpha$ photons as a result of atomic cascades (Hirata 2006; Pritchard \& Furlanetto 2006). When the coupling to the gas temperature is negligible (e.g. $\left.x_{\text {tot }} \sim 0\right), T_{\mathrm{S}} \sim$ $T_{\gamma}$ and there is no signal. On the other hand, for large $x_{\mathrm{tot}}, T_{\mathrm{S}}$ simply follows $T_{K}$.

\subsection{Simulations}

We use the code SimFast $21^{8}$ described in Santos et al. (2010) to calculate the spin temperature and the corresponding $21 \mathrm{~cm}$ signal. The code starts by calculating the linear density field in a box followed by the corresponding halo and velocity fields. Then, it computes the nonlinear density and halo fields, the collapsed mass distribution, the corresponding star formation rate, the IGM gas temperature, the Ly $\alpha$ coupling, the collisional coupling and finally the $21 \mathrm{~cm}$ brightness temperature accounting for all the contributions including the correction due to redshift space distortions using the nonlinear velocity field. In order to probe the full range of $k$ space, we generated two end to end simulations using the SimFast 21 code, one large simulation with $1 \mathrm{Gpc}$ in size and $1.6 \mathrm{Mpc}$ resolution (S1) and another with higher resolution $(0.186 \mathrm{Mpc})$ but smaller $143 \mathrm{Mpc}$ in size (simulation $\mathrm{S} 2$ ). This code has the advantage of being modular, allowing to replace some of the steps by simulation boxes generated by other techniques. Therefore, for comparison, we also applied our $\operatorname{Ly} \alpha$ calculation to the output (star formation rate, matter density) from the Trac et al. (2008) simulation, thus providing a check of the approximation used to generate the halo mass function. We call this simulation of the $21 \mathrm{~cm}$ signal, S3, with a size of $143 \mathrm{Mpc}$ and a resolution of $0.186 \mathrm{Mpc}$. Table 1 shows a summary of the simulations used. All simulations use halos with masses down to $10^{8} M_{\odot}$ corresponding to a minimum virial temperature of $T \sim 10^{4} \mathrm{~K}$. Simulation $\mathrm{S} 1$ cannot resolve halos down to these mass scales and we populate the remaining halos in each (empty) cell using a Poisson sampling biased with the underlying density field, giving a mass function consistent with

\footnotetext{
${ }^{8}$ Available at http://simfast21.org.
} 
$N$-body simulations once non-linear corrections are applied, as described in Santos et al. (2010). With the new version of the code, we also allow a hybrid approach instead of the "Poisson" method, where, after the halo finding algorithm, we calculate the unresolved collapsed mass in each empty cell using the collapsed fraction $f_{\text {coll }}$ from the extended Press-Schechter formula (see e.g. Zahn et al. 2007). This is fine as long as we do not want to resolve halos within a cell. Note that this is used at the cell level, allowing to also apply the non-linear corrections to each cell. Both approaches give similar results in terms of the $21 \mathrm{~cm}$ signal, although the later method can be faster as we decrease the halo minimum mass used in the simulation.

Simulation S3 was run using a hybrid simulation code in modeling cosmic reionization (Trac et al. 2008), incorporating $\mathrm{N}$-body, hydrodynamic, and RT algorithms to solve the coupled evolution of the dark matter, baryons, and radiation (Trac \& Pen 2004, 2006; Trac \& Cen 2007). It covers a large dynamic range and satisfies the requirements of having sufficiently high resolution to capture small-scale structure, and simultaneously a sufficiently large volume to reduce sample variance (Barkana \& Loeb 2004). The simulation is run in two steps. The first step involves running a high-resolution $N$-body simulation with $3072^{3}$ dark matter particles on an effective mesh with $11520^{3}$ cells in a comoving box, $143 \mathrm{Mpc}$ on a side. We identified collapsed dark matter halos on the fly using a friends-of-friends algorithm, with a linking length $b=0.2$ times the mean inter-particle spacing, in order to model radiation sources and sinks. With a particle mass resolution of $2.68 \times 10^{6} h^{-1} M_{\odot}$, we can reliably locate all dark matter halos with virial temperatures above the atomic cooling limit $\left(T \sim 10^{4} \mathrm{~K}\right)$ with a minimum of $\sim 40$ particles (Heitmann et al. 2008), and half of this collapsed mass budget is resolved with $>400$ particles per halo. Our halo mass functions are in very good agreement with other recent work (e.g. Reed et al. 2007; Lukic et al. 2007; Cohn \& White 2008). The second step produces a hydrodynamic + RT simulation with moderate resolution, but incorporating subgrid physics modeled using the high-resolution information from the large $N$-body simulation. Radiation sources are prescribed and star formation rates calculated using the halo model described in Trac \& Cen (2007). We consider only Population II stars from starbursts (Schaerer 2003a) as contributing to the ionizing photon budget. The hydro+RT simulation utilizes equal numbers $\left(N=1536^{3}\right)$ of dark matter particles, gas cells, and adaptive rays, where for the latter, we track 5 frequencies above the hydrogen ionizing threshold of $13.6 \mathrm{eV}$. The photo-ionization and photo-heating rates for each cell are calculated from the incident radiation flux and used in the non-equilibrium solvers for the ionization and energy equations. The initial conditions are generated with a common white noise field and a linear transfer function calculated with CAMB (Lewis et al. 2000).

In order to calculate the Ly $\alpha$ coupling in a given cell, the algorithm assumes that the scattering rate is proportional to the total Lyman series flux arriving in that cell (see again Santos et al. 2010). This Lyman series flux is obtained through a 3-dimensional integration of the comoving photon emissivity, $\epsilon(\mathbf{x}, v, z)$ (defined as the number of photons emitted at position $\mathbf{x}$, redshift $z$ and frequency $v$ per comoving volume, per proper time and frequency), which is assumed proportional to the star formation rate:

$\epsilon_{\alpha}(\mathbf{x}, v, z)=\operatorname{SFRD}(\mathbf{x}, z) \epsilon_{\mathrm{b}}(v)$,

where $\operatorname{SFRD}(\mathbf{x}, z)$ is the star formation rate density from the simulation (in terms of the number of baryons in stars per comoving volume and proper time). As stated above, in the simulation S3 we used the star formation rate obtained from the Trac et al. (2008) simulation. $\epsilon_{\mathrm{b}}(v)$ is the spectral distribution function of the sources (defined as the number of photons per unit frequency emitted at $v$ per baryon in stars). We assumed a power law model for $\epsilon_{\mathrm{b}}(v)$ :

$\epsilon_{\mathrm{b}}(v)=A v^{\alpha}$,

between $v_{\alpha}(10.2 \mathrm{eV})$ and the Lyman limit frequency $(13.6 \mathrm{eV})$. This will allow us to calculate how sensitive the $21 \mathrm{~cm}$ power spectrum is to changes in the parameters of the emission model.

We take $\alpha=-0.9$ and $A$ such that the integration between the Ly $\alpha$ and Lyman limit frequencies gives a total emission of 20000 photons per baryon. These numbers are based on the expected PopII spectra from Schaerer (2003b). Note that the parameter $A$ will be totally degenerate with the star formation rate efficiency in our model. Since the initial mass function (IMF) is likely to be dominated by massive stars, this single power law model is likely to be a good description of the emission spectra (see e.g. Leitherer et al. 1999), although a broken power law can sometimes provide a slightly better fit (Pritchard \& Furlanetto 2006). Moreover, as we shall see later, results are very insensitive to $\alpha$, essentially depending on the total number of photons emitted per baryon. We also note that the above calculation of Ly $\alpha$ radiation does not take into account full radiative transfer effects via their scattering off of neutral hydrogen atoms (scattering in the wings of the Ly $\alpha$ line, Chuzhoy \& Zheng 2007; Semelin et al. 2007, could increase the amplitude of the fluctuations on small scales). As a result, we expect that some details of the signal on small scales due to $\operatorname{Ly} \alpha$ coupling to be partly inaccurate.

Since the details of the galaxy emissivity depend upon the assumed initial mass function (IMF), which is poorly known (Barkana \& Loeb 2001), these values are subject to considerable uncertainty. Constraints would therefore provide useful information about the properties of the first galaxies. These uncertainties make the mapping between the mean value of the Ly $\alpha$ coupling $\left\langle x_{\alpha}\right\rangle$ and redshift uncertain. We will therefore describe the shape of the Ly $\alpha$ power spectrum for a given $\left\langle x_{\alpha}\right\rangle$ and caution that the redshift at which that actually occurs may be very different from our fiducial model.

\subsection{Comparison}

The $21 \mathrm{~cm}$ signal occurs over a wide range of scales, with Ly $\alpha$ fluctuations contributing at wave-numbers $0.01 \lesssim k /(h / \mathrm{Mpc}) \lesssim$ 10. Since none of our simulations can cover this broad set of scales individually, we exploit the modularity of the code to piece together two simulations to cover the full range. To check that this gives self-consistent results, in Fig. 1 we plot the full $21 \mathrm{~cm}$ brightness temperature for the three simulations at a few values of the mean Ly $\alpha$ coupling $\left\langle x_{\alpha}\right\rangle$. In each case, we take into account fluctuations from the density, velocity and Ly $\alpha$ terms as well as the collisional coupling, ionization fraction (although at high redshifts this is negligible) and gas temperature (note again that for simulation S3 we calculate the temperature using our semi-numerical code based on the simulation SFRD). We see a smooth transition from the $\mathrm{S} 1$ to the $\mathrm{S} 2$ simulation, showing for the first time, the full range of scales from $k \sim 0.01 \mathrm{~h} / \mathrm{Mpc}$ to $k \sim 20 \mathrm{~h} / \mathrm{Mpc}$ with an amplitude that is roughly between $100 \mathrm{mK}^{2}$ and $1000 \mathrm{mK}^{2}$ on the range of interest, which should help the detection of the signal. The increase in the amplitude as the coupling increases is due primarily to the cooling of the IGM gas in this redshift range, which leads to a stronger absorption signal. Note that, as explained in Santos et al. (2010), when 


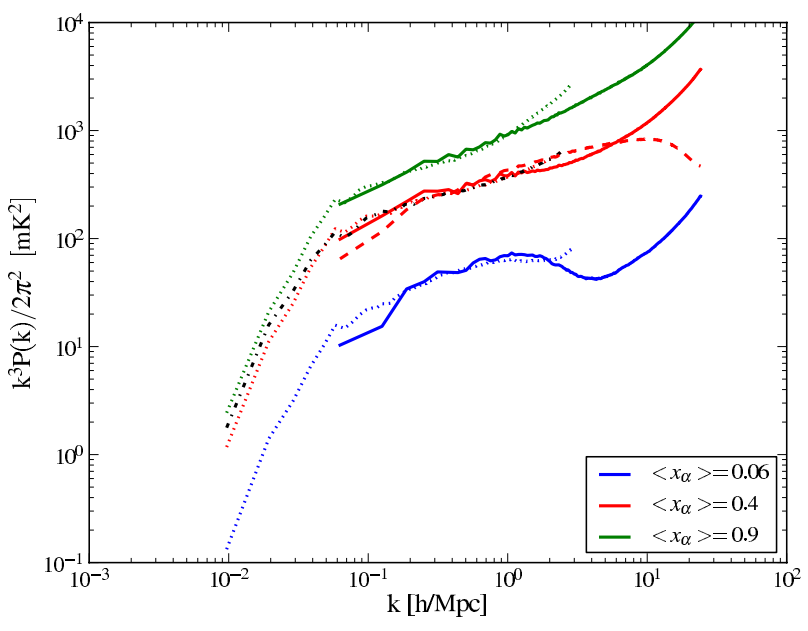

Fig. 1. The $21 \mathrm{~cm}$ brightness temperature with all fluctuations included. Dotted - simulation S1, solid - simulation S2, dashed - simulation S3. Redshifts from top to bottom are: $19.25\left(\left\langle x_{\alpha}\right\rangle=0.9\right), 20.25\left(\left\langle x_{\alpha}\right\rangle=0.4\right)$, $22.5\left(\left\langle x_{\alpha}\right\rangle=0.06\right)$. The black dash-dotted line shows the correction for $\left\langle x_{\alpha}\right\rangle=0.4$ when doing the full $\operatorname{Ly} \alpha$ flux inhomogeneous integration.

integrating the Ly $\alpha$ flux, we assume the star formation rate is homogeneous above a certain large scale in order to make the calculation faster. In this paper this scale was set to $143 \mathrm{Mpc}$ which is the origin of the slight break we see in Fig. 1 for $x_{\alpha} \gtrsim 1$. The black dash-dotted line in this figure shows the correction for $x_{\alpha}=0.4$ if instead we did the inhomogeneous integration up to $300 \mathrm{Mpc}$. Since this is a small correction, we decided to keep the limit to $143 \mathrm{Mpc}$.

For comparison, the dashed line shows the power spectrum from simulation S3, which agrees quite well with results using the semi-numerical code validating our halo/star formation prescription (although in S3 there is a decrease of the signal on very small scales, which is related to a smoothing of the velocity term on these scales). An extensive comparison made by Baek et al. (2010) using radiative transfer simulations (although for smaller box size $\sim 100 \mathrm{Mpc} / \mathrm{h}$ and lower mass resolution $\gtrsim 10^{10} M_{\odot}$ ) shows a similar spectrum for models in the same parameter range.

Since it will be the focus of the present paper, we now examine the Ly $\alpha$ fluctuations in more detail. In Fig. 2 we show the power spectrum of the quantity $A_{\alpha} \equiv \frac{x_{\alpha}}{1+x_{\alpha}}$ for the three simulations at four different values of $\left\langle x_{\alpha}\right\rangle . A_{\alpha}$ represents the contribution of the Ly $\alpha$ coupling to the $21 \mathrm{~cm}$ signal and varies between 0 and 1 . Note that the $x_{\alpha}$ field tends to be dominated by the large values close to individual sources, which obscures the details of the coupling since in these regions $A_{\alpha}$ saturates. The amplitude of the power spectrum principally depends on $\left\langle x_{\alpha}\right\rangle$ with the fluctuations peaking at $\left\langle x_{\alpha}\right\rangle \sim 0.5$, suggesting that this is the regime to best extract information related to the Wouthuysen-Field effect $(z \sim 20$ in our model), and we will concentrate on these redshifts from now on. Figure 2 shows again the correction to the calculation if we do not impose a limit to the Ly $\alpha$ inhomogeneous integration (top, cyan dashed line, for $x_{\alpha}=0.9$ ). This correction will be more evident as $x_{\alpha}$ increases (see the "break" for $x_{\alpha}=8.4$ ) but at the same time the contribution to the overall $21 \mathrm{~cm}$ signal will become smaller so that it should be safe to neglect this correction.

Comparing to Fig. 14 in Baek et al. (2009) we see that the fluctuation level is similar, although we do not see their small scale increase in power due to point sources for $k>1 \mathrm{Mpc} / \mathrm{h}$. The difference on small scales $(k>1 \mathrm{Mpc} / \mathrm{h})$ between our

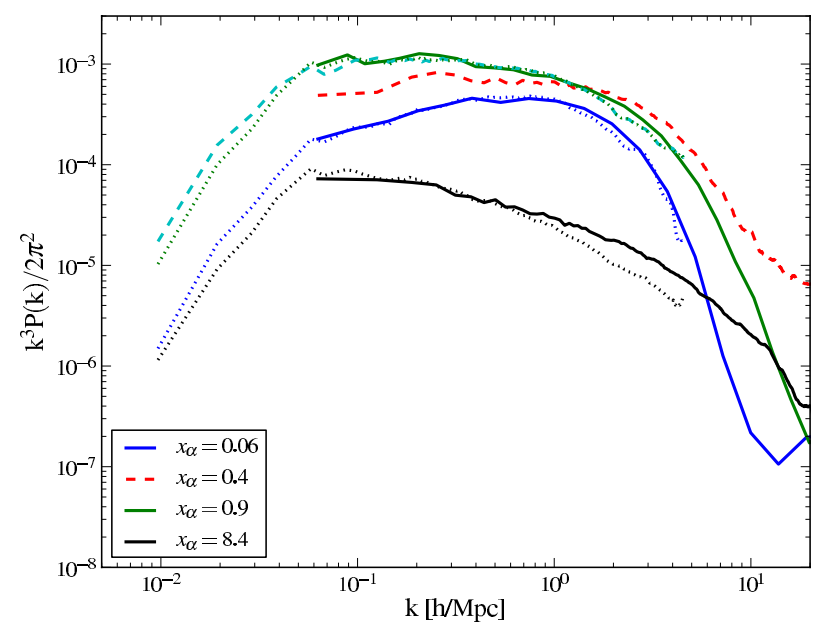

Fig. 2. Power spectrum of $\frac{x_{\alpha}}{1+x_{\alpha}}$ for several values of $\left\langle x_{\alpha}\right\rangle$. Dotted lines correspond to simulation $\mathrm{S} 1$, solid lines to simulation $\mathrm{S} 2$ and the top dashed line to simulation S3. Redshifts from top to bottom are: 19.25 $\left(\left\langle x_{\alpha}\right\rangle=0.9\right), 20.25\left(\left\langle x_{\alpha}\right\rangle=0.4\right), 22.5\left(\left\langle x_{\alpha}\right\rangle=0.06\right), 18.75\left(\left\langle x_{\alpha}\right\rangle=8.4\right)$. Again, the top, cyan dashed line shows the correction when doing the full calculation.

results and those of (Baek et al. 2009) is, at least in part, likely due to lack of Ly $\alpha$ radiative transfer treatment in ours (although differences in size and mass resolution of the simulations should also be considered). As will become clear later, it is the $21 \mathrm{~cm}$ observations at large scales that offer the best means to extract Ly $\alpha$ physics, so the inaccuracies at small scales do not pose a significant problem.

The full $N$-body simulation (S3) agrees reasonable well with our calculation and again we see a smooth transition from the very large scale simulation to the high resolution one, giving us a complete view over all scales of the expected signal. Although it is clear that there is room for more detailed numerical work on the $21 \mathrm{~cm}$ signal, these comparisons give us confidence in the semi-analytic simulation and we now turn to exploring the consequences for observations.

\subsection{Identifying the Ly $\alpha$ epoch}

In principle, there are several contributions to the $21 \mathrm{~cm}$ brightness temperature and full modeling of the signal would be required to disentangle the part due to Ly $\alpha$ fluctuations and obtain parameter constraints on the first galaxies. However, as we can see from Figs. 3 and 4, both the fluctuations in the collisional coupling and in the gas temperature should be subdominant at the redshifts where the Ly $\alpha$ fluctuations are strongest (when $\left\langle x_{\alpha}\right\rangle<1$ and $k<1 h / \mathrm{Mpc}$ ) making the analysis of the signal more straightforward (the ionization is very small at these redshifts, so that their contribution to the fluctuation power is completely negligible). The fluctuations in the gas temperature shown in Fig. 4 only include the heating due to X-rays, which can potentially be harder to separate from the Ly $\alpha$ fluctuations. On top of this we also have fluctuations in the gas temperature due to the adiabatic cooling process which are essentially proportional to the matter density perturbations. Both contributions are intrinsically included in the simulation, although, as we see, the effect from $\mathrm{x}$-ray heating is negligible.

The question then is if whether we can actually identify this epoch from observations rather than theoretical guesses. In many models, the global (average) $21 \mathrm{~cm}$ signal clearly shows the transition from Ly $\alpha$ coupling to X-ray heating and finally 


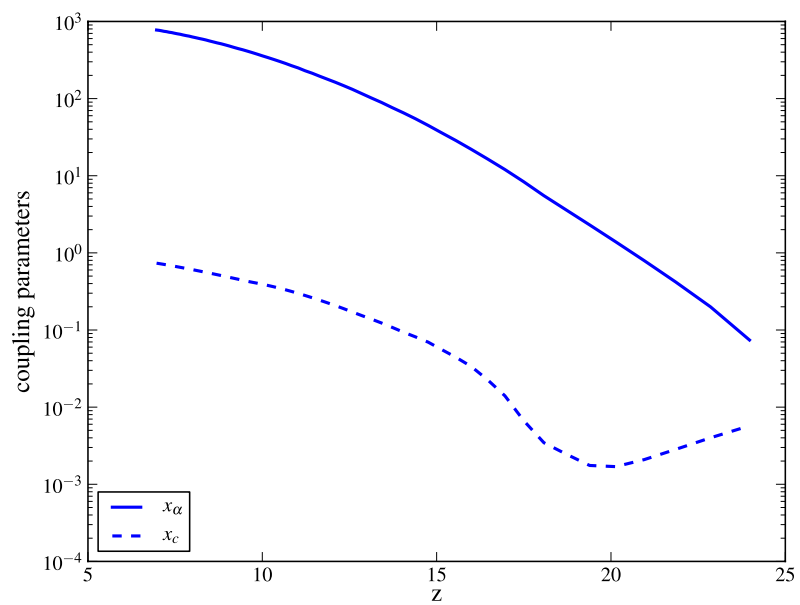

Fig. 3. The average $x_{\alpha}$ and $x_{\mathrm{c}}$ coupling parameters as a function of redshift for the S2 simulation. The Ly $\alpha$ coupling dominates over collisions at all redshifts considered.

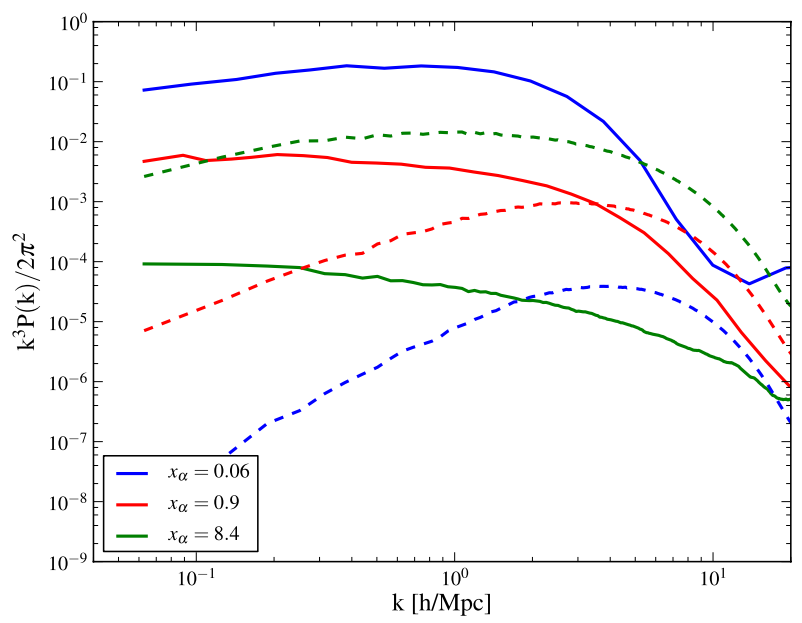

Fig. 4. The fluctuations from $\operatorname{Ly} \alpha, A_{\alpha}$ (solid) and the gas temperature, $1-\frac{T_{\gamma}}{T_{K}}$ (dashed), contributing to the $21 \mathrm{~cm}$ brightness temperature. Note that both quantities are normalized and the gas temperature fluctuations only include the $\mathrm{x}$-ray heating.

to emission once the gas is heated above the CMB temperature (Pritchard \& Loeb 2010). This average signal will not be accessible to interferometers, but as was shown in Santos et al. (2008), Fig. 9, the large scale $21 \mathrm{~cm}$ power spectrum can also depict the evolution of the signal.

The rms of the signal $\left(\left\langle\left(\delta T_{\mathrm{b}}-\left\langle\delta T_{\mathrm{b}}\right\rangle\right)^{2}\right\rangle\right)$ can also provide that information and should be easier to measure with interferometers. As we can see in Fig. 5, at $z>25$ the signal is initially small since coupling to the gas temperature is low. As the Ly $\alpha$ coupling increases, the spin temperature approaches the gas temperature and the rms of the signal increases, not only due to fluctuations on the Ly $\alpha$ field but also because the factor $A_{\alpha}\left(1-T_{\gamma} / T_{K}\right)$ is increasing (in absolute terms) since the gas is still cooling adiabatically. Once the gas temperature begins to increase due to $\mathrm{X}$-ray heating the signal decreases and we see the turning point at $z \sim 16$ (the transition from absorption to emission occurs later at $z \sim 13$. Finally, the gas is heated far above the CMB temperature and the signal plateaus until reionization finally causes it to die away at $z \sim 7$.

Note that as X-rays start heating the IGM, the signal does not drop immediately since this heating is inhomogeneous and

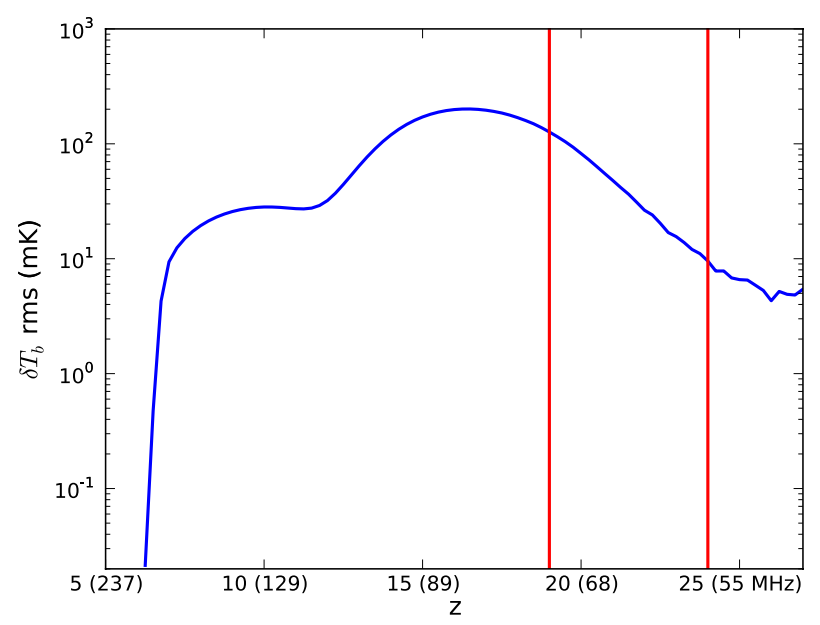

Fig. 5. $\sqrt{\left\langle\left(\delta T_{\mathrm{b}}-\left\langle\delta T_{\mathrm{b}}\right\rangle\right)^{2}\right\rangle}$ (rms) of the signal as a function of redshift for simulation S2 (frequency in parenthesis). Red lines indicate the region where we can safely ignore fluctuations from collisions and X-ray heating.

there will be some region still cooling adiabatically as well as temperature fluctuations contributing to the rms. Therefore we see a small plateau at the peak of the signal starting at $z \sim 19$. The conclusion is that it should be safe to neglect the contributions of the X-ray heating to the $21 \mathrm{~cm}$ signal between the points when the rms (or the average) starts rising at $z \gtrsim 24$ and before we reach the plateau at the maximum $z \lesssim 19\left(x_{\alpha} \sim 0.9\right)$, at least on large scales where the $\operatorname{Ly} \alpha$ contribution dominates over the temperature as we can see in Fig. 4.

In this case, the equation simplifies and we can write the full $21 \mathrm{~cm}$ signal as:

$\delta T_{\mathrm{b}}(v)=C(z)(1+\delta)\left(1+\delta_{A_{\alpha}}\right)\left(1+\delta_{A_{v}}\right)(1+\beta \delta)$,

where $\beta=2 / 3 \bar{T}_{K} /\left(\bar{T}_{K}-T_{\gamma}\right)$ (note the different definition from Barkana \& Loeb 2005b), corresponding to the adiabatic cooling process $\left(T_{K} \propto \rho^{2 / 3}\right)$ and $\bar{T}_{K} \approx 180\left(\frac{1+z}{100}\right)^{2} \mathrm{~K}$. Also,

$$
\begin{aligned}
C(z) \approx & 23\left(\frac{0.7}{h}\right)\left(\frac{\Omega_{\mathrm{b}} h^{2}}{0.02}\right)\left[\left(\frac{0.15}{\Omega_{\mathrm{m}} h^{2}}\right)\left(\frac{1+z}{10}\right)\right]^{1 / 2} \\
& \times\left\langle 1-\frac{T_{\gamma}}{T_{K}}\right\rangle\left\langle A_{\alpha}\right\rangle\left\langle A_{v}\right\rangle \mathrm{mK}
\end{aligned}
$$

with $A_{v}=\left(\frac{1}{1+1 / H \mathrm{~d} v_{r} / \mathrm{d} r}\right)\left(\left\langle A_{v}\right\rangle \sim 1\right)$. Figure 6 shows the contribution to the full $21 \mathrm{~cm}$ signal from each of the terms considered on Eq. (5) using simulations S1 and S2. Again we see that the Ly $\alpha$ term dominates over large scales $k<1 \mathrm{~h} / \mathrm{Mpc}$, which makes it all important to be able to generate large volume simulations $(>100 \mathrm{Mpc} / \mathrm{h}$ ) and give us confidence that it should be possible to extract the $\operatorname{Ly} \alpha$ signal over the redshift range proposed without confusion from other contributions. Moreover, the small contribution at these scales from the other terms in Eq. (5) can be modeled with reasonable accuracy if we know the matter power spectrum.

\section{Measurements with the SKA}

In this section, we outline details related to the experimental measurement of the $21 \mathrm{~cm}$ signal at the very high redshifts during the epoch where Ly $\alpha$ dominates. 


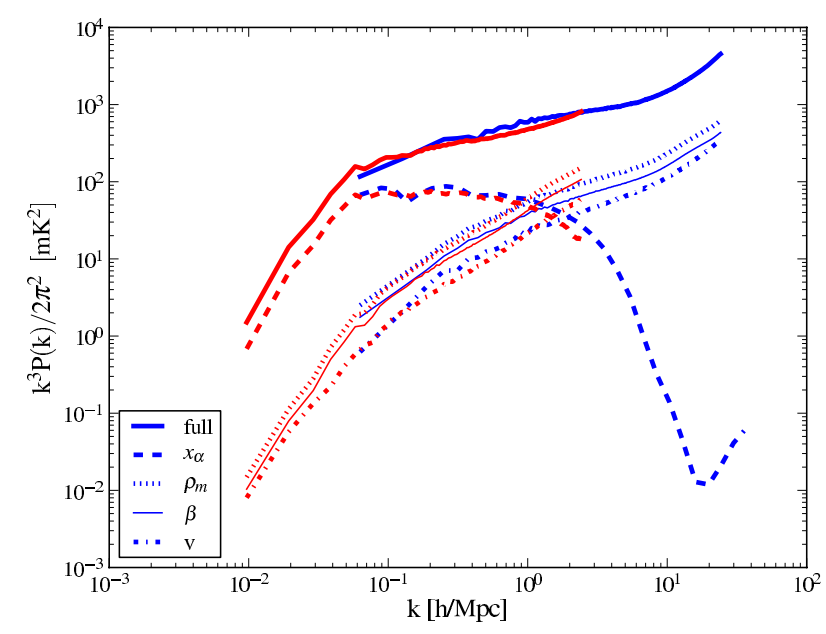

Fig. 6. The $21 \mathrm{~cm}$ power spectrum and its main contributions at $\left\langle x_{\alpha}\right\rangle=$ 0.4 ( $z \sim 20.25)$ using simulation $S 1$ (red) and simulation S2 (blue). Top solid lines - all contributions included; dashed - Ly $\alpha$ only; dotted - matter density; bottom thin solid lines - fluctuations on the gas temperature from adiabatic cooling only; dot-dashed - velocity fluctuations only.

\subsection{Noise power spectrum}

Before we go into the details of the experimental setup, we quickly review the noise calculation that goes into our analysis. Following Bowman et al. (2006); Mao et al. (2008); McQuinn et al. (2006), the expected error $\Delta P(k, \theta)$ on the measurement of the 3-d power spectrum of the signal $P_{\mathrm{S}}(k, \theta)$ with noise $P_{N}(k, \theta)$ is given by

$\Delta P(k, \theta)=\frac{1}{\sqrt{N_{\mathrm{m}}(k, \theta)}}\left[P_{\mathrm{S}}(k, \theta)+P_{N}(k, \theta)\right]$,

where we are assuming that the power spectrum depends only on the moduli $(k)$ of the vector $\boldsymbol{k}$ and on the angle $\theta$ between $\boldsymbol{k}$ and the line of sight. $N_{\mathrm{m}}(k, \theta)$ is the total number of modes in $\boldsymbol{k}$ space contributing to the measurement (note that the sum is only done over half the sphere). In order to obtain the above expression, we "grid" the $\boldsymbol{k}$ space into pixels of size $\mathrm{d} k_{\perp}^{2} d k_{\|}$, where $\perp$ denotes the component perpendicular to the line of sight and \| the parallel one and assume that measurements in different pixels are uncorrelated. The size of these pixels is just determined by the real space volume of the observed region:

$\mathrm{Vol}=r^{2} y A_{\mathrm{FoV}} B$,

where $A_{\mathrm{FoV}}$ is the field of view of the experiment (in radians), $B$ is the bandwidth for this particular measurement, $r(z)=$ $\int_{0}^{z} c H^{-1} \mathrm{~d} z^{\prime}$ is the comoving distance to redshift $z$ and $y \equiv$ $\lambda_{21}(1+z)^{2} / H(z)$ is a conversion factor between frequency intervals and comoving distances $(H(z)$ is the Hubble expansion rate and $\lambda_{21} \approx 21.1 \mathrm{~cm}$ the rest frame wavelength of the $21 \mathrm{~cm}$ line). We can relate $\mathrm{d} k_{\perp}$ to the resolution in the $u-v$ (visibility) space, $\mathrm{d} u$ (the Fourier dual of the angular coordinates on the sky) by $\mathrm{d} k_{\perp}=2 \pi \mathrm{d} u / r$ (using our Fourier conventions). Note that

$\mathrm{d} u=1 / \sqrt{A_{\mathrm{FoV}}}$

and is related to the effective collecting area of one element of the interferometer, $A_{\mathrm{e}}=\lambda^{2} \mathrm{~d} u^{2}$, where $\lambda$ is the wavelength of observation (but care must be taken in this relation due to multibeaming). The resolution along the line of sight is just $\mathrm{d} k_{\|}=$ $2 \pi /(\mathrm{yB})$ (visibilities are Fourier transformed along the frequency coordinate).
Finally, the noise power spectrum is given by

$P_{N}(k, \theta)=r^{2} y \frac{T_{\text {sys }}^{2}}{\mathrm{~d} u^{2} t(k, \theta)}$,

where $t(k, \theta)$ is the time spent observing a given $\boldsymbol{k}$ pixel which is just the time spent observing the corresponding pixel of resolution $\mathrm{d} u^{2}$ on the $u-v$ plane, $\mathbf{u}=r \boldsymbol{k}_{\perp} /(2 \pi)$. For a total observation time, $t_{0}$, this will be related to the baseline density distribution, $n(|\mathbf{u}|)$, through $t(k, \theta)=t_{0} \mathrm{~d} u^{2} n(|\mathbf{u}|)=t_{0} \mathrm{~d} u^{2} n(r k \sin (\theta) /(2 \pi))$ assuming the baseline density is rotationally invariant (which is a good approximation due to Earth rotation). If we make the further assumption that the baseline density distribution is constant on the $u-v$ plane up to a maximum baseline $D_{\max }$ (which is not the same as assuming an uniform distribution of antennas), we have

$n(|\mathbf{u}|)=\frac{\lambda^{2} N_{a}^{2}}{\pi D_{\max }^{2}}$,

noting that the integration over half the plane should give approximately $N_{a}^{2} / 2\left(N_{a}\right.$ is the number of elements in the interferometer). The noise power spectrum then further simplifies to:

$P_{N}(k, \theta)=r^{2} y \frac{\pi \lambda^{2} D_{\text {max }}^{2} T_{\text {sys }}^{2}}{t_{0} A_{\text {tot }}^{2}}$,

where $A_{\text {tot }}$ is the total collecting area of the telescope. The system temperature was modeled by

$T_{\text {sys }}(z)=50+60\left(\frac{1+z}{4.73}\right)^{2.55} \mathrm{~K}$

where the first term is the receiver noise temperature (assumed to be $50 \mathrm{~K}$ ) and the second term is the much larger sky temperature which is dominated by the Galactic Synchrotron at the redshifts of interest. Note in particular that neither the bandwidth or the frequency resolution show up in the noise calculation (they can be used instead to increase the number of modes $N_{\mathrm{m}}$ available to reduce the total error). Moreover, looking at the last expression we see that $P_{N}$ decrease as $1 / A_{\text {tot }}^{2}$ once we fix $t_{0}$ and the maximum baseline. Once we achieve $P_{\mathrm{S}} \sim P_{N}$ the only way to decrease the total error is through $N_{\mathrm{m}}$ which does not depend on the total collecting area or the baseline distribution.

\subsection{Experimental setup}

The current generation of radio-interferometers now in construction such as the MWA and LOFAR will not have the collecting area necessary to probe the high redshift universe $(z>$ 15). This will require a second generation experiment such as the SKA, with an observational window between $70 \mathrm{MHz}$ and $10 \mathrm{GHz}$ and planned to be completed by 2020 . The SKA will probably be made up of three different instruments (Schilizzi et al. 2007; Faulkner 2010): SKA-low, tailored for the low frequency signal between $70 \mathrm{MHz}$ to $450 \mathrm{MHz}$, probably made up of sparse Aperture Arrays so that the collecting area scales as $\lambda^{2}$; SKA-mid between $400 \mathrm{MHz}$ and $1.4 \mathrm{GHz}$, using dense Aperture Arrays; and SKA-high, using dishes for frequencies above $1.2 \mathrm{GHz}$ (note the small overlap between ranges). In this paper, we will assume a low frequency "SKA type" experiment with a setup capable of probing the high redshift, prereionization epoch.

The analysis in the previous Section showed that in order to measure the imprint of the first galaxies on the $21 \mathrm{~cm}$ signal, we need to observe at least up to $z=20$, corresponding to 
a frequency of $v \sim 68 \mathrm{MHz}$. Allowing for some flexibility, we consider an experiment that would be able to measure frequencies down to $60 \mathrm{MHz}$. Taking into account the design reference for SKA, we assumed a sensitivity of $4000 \mathrm{~m}^{2} / \mathrm{K}$ at $70 \mathrm{MHz}$ (which in fact requires a total collecting area of $\sim 14 \mathrm{~km}^{2}$, well above the $1 \mathrm{~km}^{2}$ used to baptize the telescope) and scaling as $\lambda^{2}$ around these frequencies. Note that the planned collecting area has been subject to some change along the years and the current design for what is called SKA Phase 1 suggests a sensitivity of $2000 \mathrm{~m}^{2} / \mathrm{K}$ instead (Garrett et al. 2010). In order to allow for designs with lower sensitivities, we also consider instruments with $20 \%$ and $10 \%$ of the total collecting area of the design reference SKA. For the distribution of antennas, the SKADS ${ }^{9}$ reference design assumes they would be collected in 250 stations of $180 \mathrm{~m}$ diameter each, with $66 \%$ in a $5 \mathrm{~km}$ diameter core and the rest along 5 spiral arms out to a $180 \mathrm{~km}$ radius.

The signal we are looking for dominates on scales $k \lesssim$ $1 \mathrm{~h} / \mathrm{Mpc}$, requiring baselines up to $\sim 10 \mathrm{~km}$ for proper sampling at the relevant frequencies, e.g. a resolution of 0.76 arcmin at $z=20\left(k_{\max \perp} \sim 1.8 \mathrm{~h} / \mathrm{Mpc}-\right.$ higher resolution can be achieved along the frequency direction although the error would be much larger since we would only have modes along the line of sight). Taking a reasonable setup, we assumed that $70 \%$ of the total collecting area would be concentrated within this core of $10 \mathrm{~km}$ in diameter. We do not use the rest of the collecting area in the noise calculation, assuming instead this would be used for point source removal and calibration. The same applies when the total collecting area is only $10 \%$ and $20 \%$ of the reference design.

For the field of view $(\mathrm{FoV})$ we used $10 \times 10 \mathrm{deg}^{2}$, which, taking $z=20$ as reference, sets a resolution of roughly $\mathrm{d} k_{\perp} \sim$ $4.5 \times 10^{-3} \mathrm{~h} / \mathrm{Mpc}$. Note that the FoV can be traded with the number of beams (in case we want to observe separate fields) and the total instantaneous bandwidth (in case we want to probe a larger redshift range). Assuming that, in the core, correlations within stations are also possible, we considered a minimum baseline of $50 \mathrm{~m}$ giving $k_{\min \perp} \sim 9 \times 10^{-3} \mathrm{~h} / \mathrm{Mpc}$ which is enough to probe the scales of interest. The frequency interval for the analysis should be chosen carefully at these frequencies since for a fixed frequency interval, $\mathrm{d} z$ increases with redshift. For instance, an interval of $8 \mathrm{MHz}$ used in previous analysis (e.g. Bowman et al. 2007) corresponds to integrating the signal over $\mathrm{d} z \sim 2.5$ which will average out important features during the period when Ly $\alpha$ fluctuations are important. On the other hand, using a small interval means we will have fewer modes along the line of sight. Taking a compromise, we assumed that each measurement was done with an interval of $4 \mathrm{MHz}$ and a resolution of $0.01 \mathrm{MHz}$, giving $\mathrm{d} k_{\|}=0.08 \mathrm{~h} / \mathrm{Mpc}$ and $k_{\max \|}=16.6 \mathrm{~h} / \mathrm{Mpc}$. Note that the total instantaneous bandwidth should be much larger than this $(\sim 50 \mathrm{MHz})$, so we can measure several redshifts at the same time. Finally, the total integration time was taken to be $1000 \mathrm{~h}$ (see Table 2 for a summary of the experimental setup).

We followed the error calculation described above for the power spectrum assuming a distribution for the antenna/stations of the telescope such that a constant baseline density is obtained. Although this is difficult to achieve in practice since the density of stations normally decreases from the center, it turns out that this configuration gives the best signal to noise in terms of the brightness temperature maps and it should therefore give a good indication of the telescope capabilities to probe the $21 \mathrm{~cm}$ signal power spectrum. A final note on foregrounds: they can be the most damaging factor at these low frequencies (together with the calibration issues raised by the ionosphere) although it

9 http://www.skads-eu.org
Table 2. Assumed experimental setup.

\begin{tabular}{cc}
\hline \hline Parameters & Values \\
\hline Min. Freq. & $60 \mathrm{MHz}$ \\
Sensitivity & $4000 \mathrm{~m}^{2} / \mathrm{K}$ \\
Max. baseline & $10 \mathrm{~km}$ \\
Min. baseline & $50 \mathrm{~m}$ \\
FoV & $100 \mathrm{deg}^{2}$ \\
Integration time & $1000 \mathrm{~h}$ \\
Freq. interval & $4 \mathrm{MHz}$ \\
Freq. resolution & $0.01 \mathrm{MHz}$ \\
\hline
\end{tabular}

Notes. We also considered $20 \%$ and $10 \%$ of this sensitivity in our analysis. Note that only $70 \%$ of the assumed collecting area is used in the core of $10 \mathrm{~km}$ diameter.

is expected that if the foregrounds are smooth enough along the frequency direction, they can be simply removed by fitting out a smooth function (Jelić et al. 2008; Morales et al. 2006; Santos et al. 2005). In this analysis we assume that foreground cleaning was applied on a region of $32 \mathrm{MHz}$ (so as to avoid edge effects on our $4 \mathrm{MHz}$ interval) and was successful on scales smaller than this $32 \mathrm{MHz}$, e.g. we ignore scales with $k<0.01 \mathrm{~h} / \mathrm{Mpc}$ (see Harker et al. 2010). Calibration issues raised by the ionosphere can also be quite damaging in particular due to the large field of view of this low frequency interferometers (Cohen \& Röttgering 2009; Matejek \& Morales 2009; Liu et al. 2010) and the situation should become more clear once we have results from the first generation of EoR experiments. In this paper we concentrate on analysing the required minimum setup for an SKA type experiment in order to make a statistical detection of the signal, neglecting for now the challenges posed by the ionosphere.

\subsection{Power spectrum constraints}

Figure 7 shows the expected error on the 3 dimensional power spectrum, $P(k)$ (integrated over $\theta$ ), assuming the above setup. Even if foreground removal affects larger $k$-modes than assumed here we still have a huge range of measurements available up to $k \sim 7 \mathrm{~h} / \mathrm{Mpc}$ and in particular, measurements are quite good on the interval where the Ly $\alpha$ contribution is more important. On very large scales it should be possible to measure the power spectrum even with only $10 \%$ of the collecting area. Sample variance dominates on large scales while noise (dashed green line) is dominating on small scales (the number of modes on small scales is limited due to the size of the maximum baselines). Note that we could redistribute the collecting area so that the noise power spectrum followed more closely the signal. The error on large scales is kept small because there are still a large number of measurements $N_{\mathrm{m}}$ due to the high resolution on the $u-v$ space from the large field of view (see Eq. (7)).

With the above measurements of the $3 \mathrm{~d}$ power spectra, we should be able to constrain some of the characteristics of the first galaxies such as their emission spectra. Although, as we have seen, the Ly $\alpha$ signal dominates on larger scales over other contributions, these constraints will be particularly strong if we fix the cosmology, which should be a reasonable assumption given the expected level of constraints from the Planck satellite.

Up to now we have been assuming a type II emission spectrum with a total of 20000 photons per baryon emitted between the Ly $\alpha$ and Lyman limit frequency. If instead we assume that the stars responsible for the Ly $\alpha$ coupling are Pop III, we should have around 5000 photons emitted per baryon used in stars with a spectral index of $\alpha=0.29$. Figure 8 shows the results of changing the emission model with the dashed curves corresponding to 


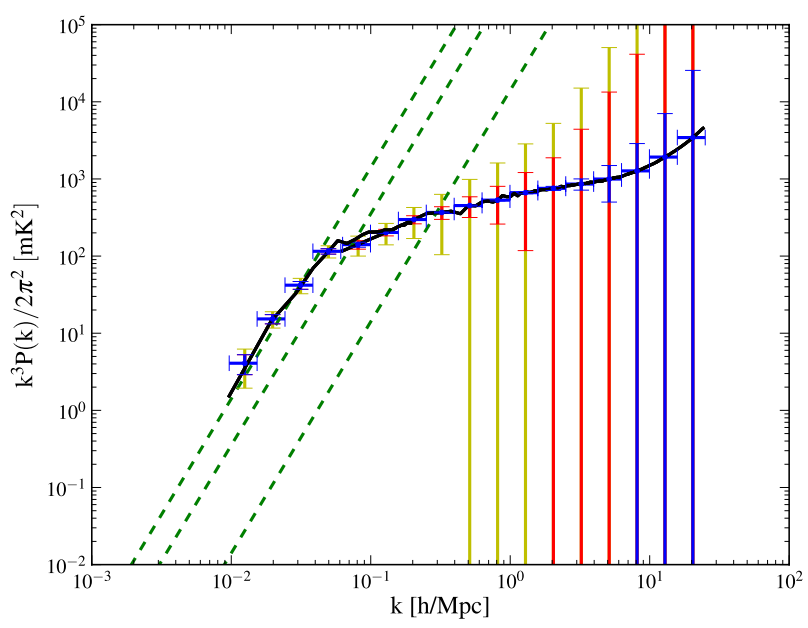

Fig. 7. Error on the $3 \mathrm{~d} 21 \mathrm{~cm}$ power spectrum at $z=20.25\left(\bar{x}_{\alpha}=0.4\right)$ assuming an SKA type experiment. Solid black - signal for simulations $\mathrm{S} 1+\mathrm{S} 2$; dashed green - noise power spectrum (equation 12) for the full SKA collecting area, 20\% and 10\% (increasing amplitude). The expected error taking into account the available number of modes (Eq. (7)) is shown with error bars in blue, red and yellow respectively. Measurement of the large scale power spectrum should be possible as long as the signal amplitude is not 100 times smaller than current value (this is controlled by $x_{\alpha} /\left(1+x_{\alpha}\right)\left(1-T_{\gamma} / T_{\mathrm{S}}\right)$ so it should be fine for $\left.x_{\alpha}>0.01\right)$. On large scales it should be possible to measure the signal even with only $10 \%$ of the collecting area.

the PopIII case (note that due to the lower number of photons, these lines also correspond to a lower $\left\langle x_{\alpha}\right\rangle$ of 0.1 . The dotted curve corresponds to changing the spectral index to $\alpha=0.29$ while maintaining the total emission to 5000 photons, which will be difficult to distinguish under the current noise expectations (the total number of emitted photons per baryon is the most relevant parameter for the $\operatorname{Ly} \alpha$ flux). Another possibility is to assume that Pop III stars are also being formed in halos with mass less than $10^{8} M_{\odot}$. The green dot-dashed line in Fig. 8 shows the effect of considering halos down to $10^{6} M_{\odot}$ from simulation S1 at the same redshift. Note that this has a much higher coupling and if we consider higher redshifts so that $\left\langle x_{\alpha}\right\rangle \sim 0.1$ the line will be closer to the dashed one.

These calculations show that it should be possible for SKA to measure $\left\langle x_{\alpha}\right\rangle$ from observations of the $21 \mathrm{~cm}$ power spectrum at $z \approx 20$. Given a detailed model and small error bars this could be measured directly from the shape of the power spectrum. More generally, if measured over only a small range of wave-numbers, the redshift dependence of $\left\langle x_{\alpha}\right\rangle$ could be extracted by looking at the redshift evolution of the amplitude and slope in a manner analogous to that suggested by Lidz et al. (2008) for reionization. Note that, although during $\operatorname{Ly} \alpha$ domination different models have similar power spectra for the same $\left\langle x_{\alpha}\right\rangle$, its evolution with redshift could be used to distinguish these models.

Measurements of $\left\langle x_{\alpha}\right\rangle$ in a number of different redshift bins would be instrumental in constructing a " $21 \mathrm{~cm}$ Madau plot" (Madau et al. 1996). The Madau plot shows the evolution of the star formation rate as a function of redshift and producing such a plot in the very early Universe would be a major accomplishment of SKA. Measurements of $\left\langle x_{\alpha}\right\rangle$ do not give this directly, since in our model $\left\langle x_{\alpha}\right\rangle$ is determined by the product of the starformation rate and the emissivity of the galaxies. Breaking this degeneracy observationally will be difficult and would lead to a plot whose absolute normalisation was uncertain, but whose shape tracked the star-formation rate (assuming no evolution of

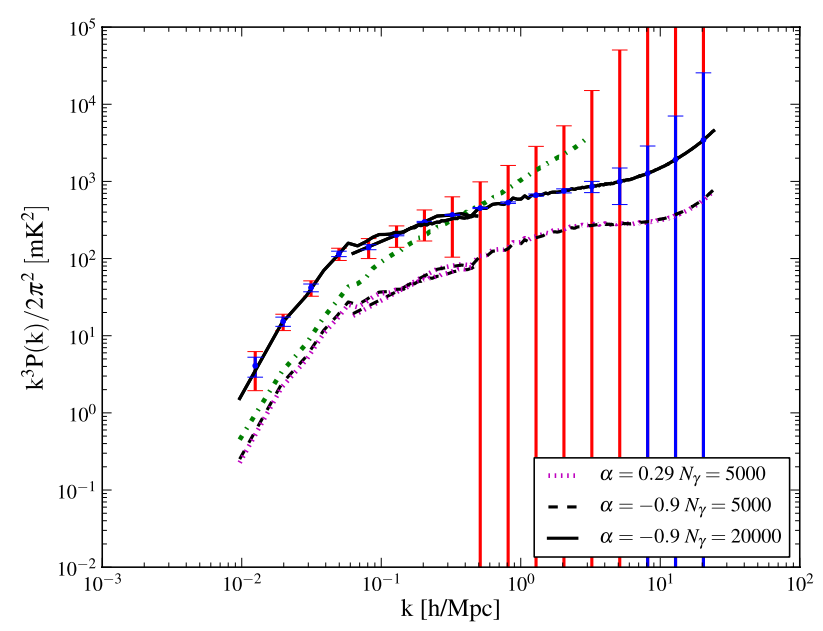

Fig. 8. $21 \mathrm{~cm}$ temperature power spectrum for a few Lyman alpha emission models and simulations S1 and S2. Top black solid lines correspond to $\left\langle x_{\alpha}\right\rangle=0.4$ while bottom dashed/dotted curves to $\left\langle x_{\alpha}\right\rangle=0.1$ (all at $z \sim 20.25$ ). The green dot-dashed line uses halos down to $10^{6} \mathrm{M}_{\odot}$ and a Pop III type emission spectra. The error bars in blue corresponds to the full SKA while the ones in red to $10 \%$ of the collecting area.

the galaxies). However, for many models of early galaxy formation (Leitherer et al. 1999; Bromm \& Larson 2004) the variation in the number of Ly $\alpha$ photons produced per baryon is only a factor of two or so. Thus with a reasonable theoretical prior a useful measure of the star-formation history could be established.

\section{Redshift space distortions}

The signal we are trying to probe is actually anisotropic due to the redshift space distortions set by the peculiar velocity of the HI gas, which is already being taken into account in Eq. (5). Using a linear approximation, $A_{\alpha} \approx \frac{\left\langle x_{\alpha}\right\rangle}{1+\left\langle x_{\alpha}\right\rangle}\left(1+\frac{1}{1+\left\langle x_{\alpha}\right\rangle} \delta_{x_{\alpha}}\right)$ and $A_{v} \approx 1-\frac{1}{H} \frac{\mathrm{d} v_{r}}{\mathrm{~d} r}$ so to first order the fluctuation in $T_{\mathrm{b}}$ becomes

$\delta T_{\mathrm{b}}(v) \approx C(z)\left(1+(1+\beta) \delta+\frac{1}{1+\left\langle x_{\alpha}\right\rangle} \delta_{x_{\alpha}}-\frac{1}{H} \frac{\mathrm{d} v_{r}}{\mathrm{~d} r}\right)$.

The peculiar velocity field originates from inhomogeneities in the density field, so that in Fourier space we can use Kaiser's approximation $\frac{1}{H} \frac{\mathrm{d} v_{r}}{\mathrm{~d} r}(\boldsymbol{k})=-\mu^{2} \delta(\boldsymbol{k})$ (Kaiser 1987) to write the velocity as a function of its dependence in $\mu=\cos (\theta)$. This linear approximation in the velocity contribution allows the brightness temperature power spectra to be separated in only three powers of $\mu$ :

$P_{T_{\mathrm{b}}}(k, \mu)=\mu^{4} P_{\mu^{4}}(k)+\mu^{2} P_{\mu^{2}}(k)+P_{\mu^{0}}(k)$.

with

$P_{\mu^{4}}(k)=C^{2}(z) P_{\delta}(k)$,

$P_{\mu^{2}}(k)=2 C^{2}(z)\left[(1+\beta) P_{\delta}(k)+\frac{1}{1+\left\langle x_{\alpha}\right\rangle} P_{\delta \delta_{x_{\alpha}}}(k)\right]$,

and

$$
\begin{aligned}
P_{\mu^{0}}(k)= & C^{2}(z)\left[(1+\beta)^{2} P_{\delta}(k)\right. \\
& \left.+\left(\frac{1}{1+\left\langle x_{\alpha}\right\rangle}\right)^{2} P_{\delta_{x_{\alpha}} \delta_{x_{\alpha}}}(k)+\frac{2(1+\beta)}{1+\left\langle x_{\alpha}\right\rangle} P_{\delta \delta_{x_{\alpha}}}(k)\right],
\end{aligned}
$$




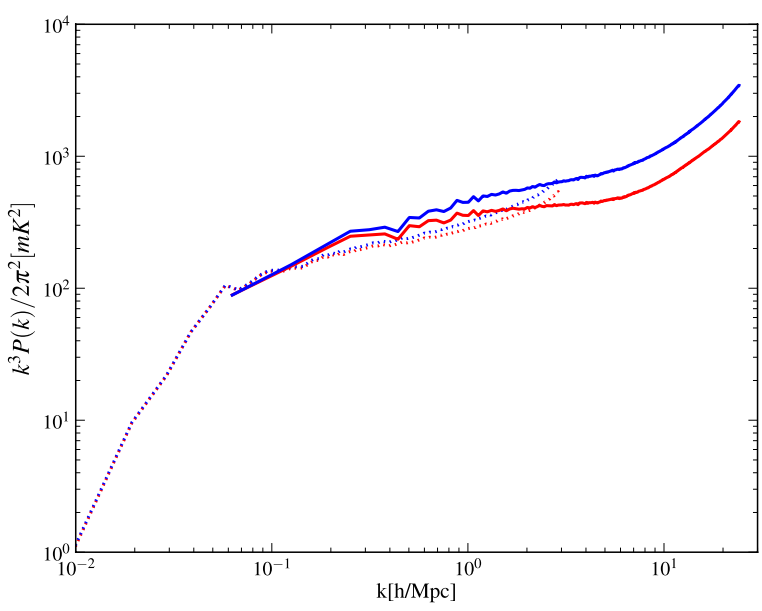

Fig. 9. Power spectra of brightness temperature fluctuations using simulation S1 (dotted lines) and using simulation S2 (solid lines) for $\left\langle x_{\alpha}\right\rangle=0.4(z \sim 20.25)$. Blue lines (top) use the exact velocity contribution and red lines (bottom) use a first order approximation in the velocity contribution.

where the power spectra $P_{a b}(k)$ for generic quantities $a$ and $b$ is defined as:

$(2 \pi)^{3} \delta_{D}^{3}\left(\boldsymbol{k}-\boldsymbol{k}^{\prime}\right) P_{a b}(k) \equiv \frac{\left\langle a(\boldsymbol{k}) b\left(\boldsymbol{k}^{\prime}\right)^{*}\right\rangle+\left\langle b(\boldsymbol{k}) a\left(\boldsymbol{k}^{\prime}\right)^{*}\right\rangle}{2}$.

This decomposition can be particularly useful at the high redshifts we are considering, where, as can be seen from Fig. 6, the contribution from the velocity compression can be relatively strong. By fitting the observed signal to the above polynomial (Eq. (15)) at each $k$, we can in principle separate the cosmological and astrophysical contributions to the brightness temperature fluctuations (Barkana \& Loeb 2005a,b). One can use $P_{\mu^{2}}$ and $P_{\mu^{0}}$ to learn about the first sources of radiation while the $P_{\mu^{4}}$ term could be used to measure the matter power spectrum directly, with no interference from any other sources of fluctuations.

\subsection{Assumptions on linearity}

The decomposition discussed in the previous section relies on assumptions of linearity in both the velocity field and the Ly $\alpha$ fluctuations. On small scales, both of these assumptions may break down and we consider both in turn. As we go to small scales, fluctuations on the density field increase and the nonlinearities in the velocity contribution can become important, so that the first order approximation to the full velocity term in Eq. (1) breaks down. Figure 9 compares the brightness temperature power spectrum from the full calculation with the case where we assume the first order approximation for the velocity. We see that for $k \gtrsim 1.0 \mathrm{~h} / \mathrm{Mpc}$ the non-linearities can become important. This result implies that for smaller scales the correct brightness temperature power spectra has a much more complicated dependence in $\mu$ than the one given by Eq. (15) which will complicate the model independent extraction of the astrophysical contributions (Shaw \& Lewis 2008).

Fluctuations in the Ly $\alpha$ background too can leave the linear regime. The information from the Ly $\alpha$ background and consequently from the physical processes that originated it, is contained in $\delta_{x_{\alpha}}$ and it can only be directly obtained from the power spectrum angular separation in powers of $\mu$ for scales where the linear approximation of $A_{\alpha}$ is valid. To show the limits where the first order approximation can be used, we plotted in Fig. 10

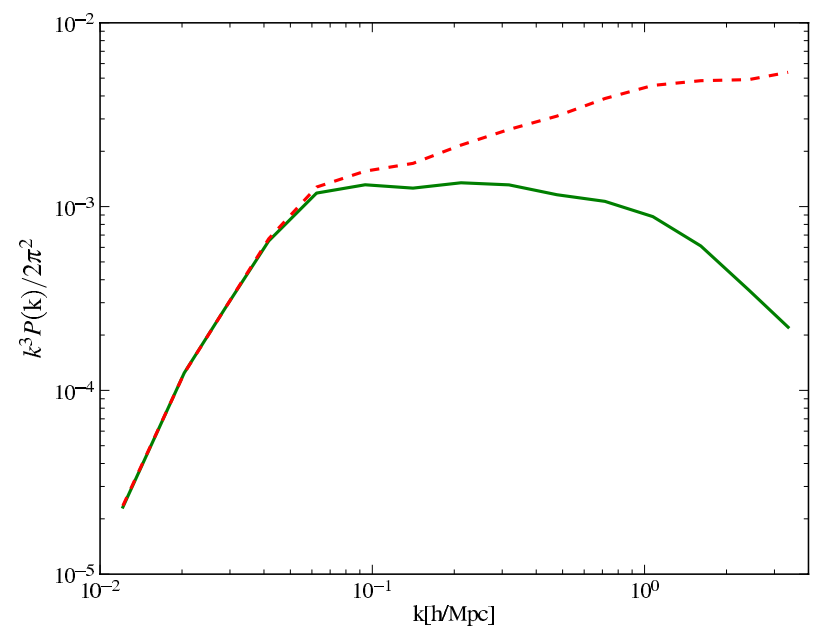

Fig. 10. Power spectra of fluctuations in $x_{\alpha} /\left(1+x_{\alpha}\right)$ built using simulation S1 for $\left\langle x_{\alpha}\right\rangle=0.4(z \sim 20.25)$. The figure shows the the total fluctuation (solid green line) and its first order approximation (dashed red line).

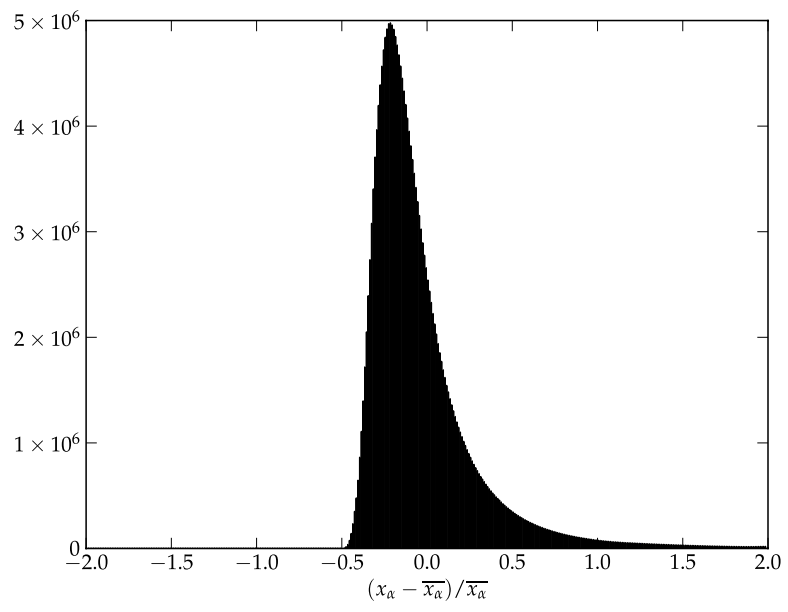

Fig. 11. Detail of the histogram of fluctuations in $\delta_{x_{\alpha}}$ using simulation S1 for $\left\langle x_{\alpha}\right\rangle=0.4(z \sim 20.25)$. The fluctuations in $\delta_{x_{\alpha}}$ go from -50 till 350 .

the power spectra of $A_{\alpha}$ compared to its linear expression, using the values from simulation $\mathrm{S} 1$. The linear approximation should be valid for $k \lesssim 0.2 \mathrm{~h} / \mathrm{Mpc}$.

Looking at the histogram of $\delta_{x_{\alpha}}$ in Fig. 11 we see that there are few points where $\delta_{x_{\alpha}}>1$. Although this number is small compared to the overall distribution (most cells in the simulation have actually $x_{\alpha} \sim 0$ at these high redshifts), they are enough to introduce non-linearities on the power spectrum calculation. This basically implies that for small scales the fluctuations in $x_{\alpha}$ will have a large dependence on the random distribution of the first galaxies so it will only be possible to do a linear approximation on $A_{\alpha}$ for scales large enough that a few small peaks of large intensity do not dominate the power spectra of the fluctuation. Some of the strong coupling regions coincide with ionized regions which may diminish the effect of strong fluctuations in the brightness temperature power spectra, however according with simulations S1, S2 and S3, at the high redshifts we are interested $(z>20)$ the ionized regions are so small that including this quantity has no meaningful effect in the linear approximation used. 


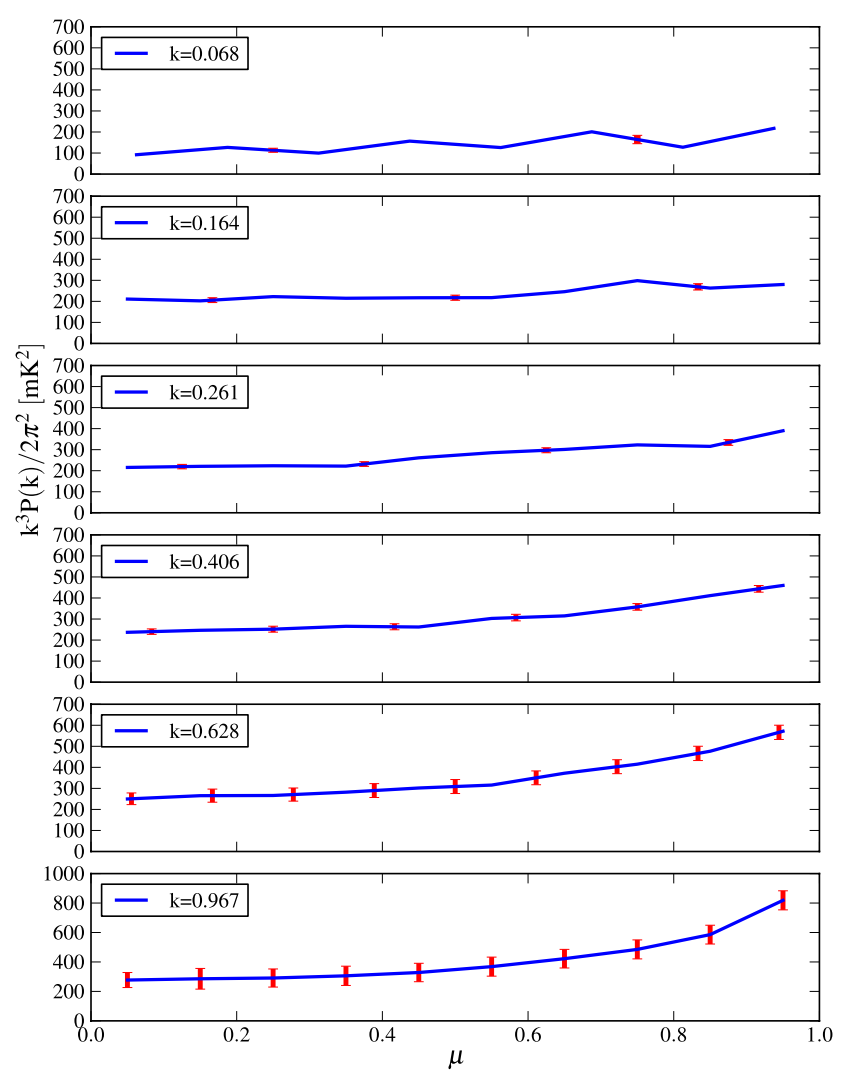

Fig. 12. Expected measurements for the SKA of the $21 \mathrm{~cm}$ power spectrum as a function of the angle with the line of sight $(z=20.25$, $\left.\bar{x}_{\alpha}=0.4\right)$.

From this study we can conclude that the brightness temperature power spectrum angular separation in Eq. (15) should be reasonable for $k<1 \mathrm{~h} / \mathrm{Mpc}$ and the linear approximations in Eqs. (17) and (18) should be valid for larger scales, $k \lessgtr$ $0.2 \mathrm{~h} / \mathrm{Mpc}$, allowing the direct extraction of information about the Ly $\alpha$ field (Barkana \& Loeb 2005b). This is fortunate since it corresponds to the scales where the Ly $\alpha$ fluctuations dominate the contributions to the brightness temperature (Fig. 6) as well as the scales where the SKA should perform better (Fig. 7). For $0.2 \lesssim k \lesssim 1.0 \mathrm{~h} / \mathrm{Mpc}$ we can still use the angular decomposition but Eqs. (17) and (18) will include higher order terms. Given the scales we are interested in, from now on we will concentrate on simulation $\mathrm{S} 1$ (1000 Mpc) which can be used to calculate the power spectrum up to $k \sim 2.3 \mathrm{~h} / \mathrm{Mpc}$.

\subsection{Constraints on $P(k, \mu)$}

As we have seen in the previous section, the redshift space distortions introduce an anisotropy in the $21 \mathrm{~cm}$ power spectrum that, in the linear regime, depends on $k$ and even powers of $\mu$. More generally non-linearities in the velocity field will introduce terms with more complicated dependence (Shaw \& Lewis 2008). Measurements of $\mathrm{P}(k, \mu)$ might provide more information on the Ly $\alpha$ signal (Barkana \& Loeb 2005a) than the spherical average $\mathrm{P}(k)$ we considered so far. Figure 12 shows the expected errors in the measurements of the $3 \mathrm{~d}$ power spectrum as a function of $\mu$, for an SKA type experiment (Table 2). The calculation follows Eq. (7) where the number of modes in each bin is now a function of $k$ and $\mu$. We used bins with a logarithmic spacing in $k$ and linear in $\mu$ (with $0 \leq \mu \leq 1$ since the power spectrum only depends on $\mu^{2}$ ). The number of modes in each bin is accounted

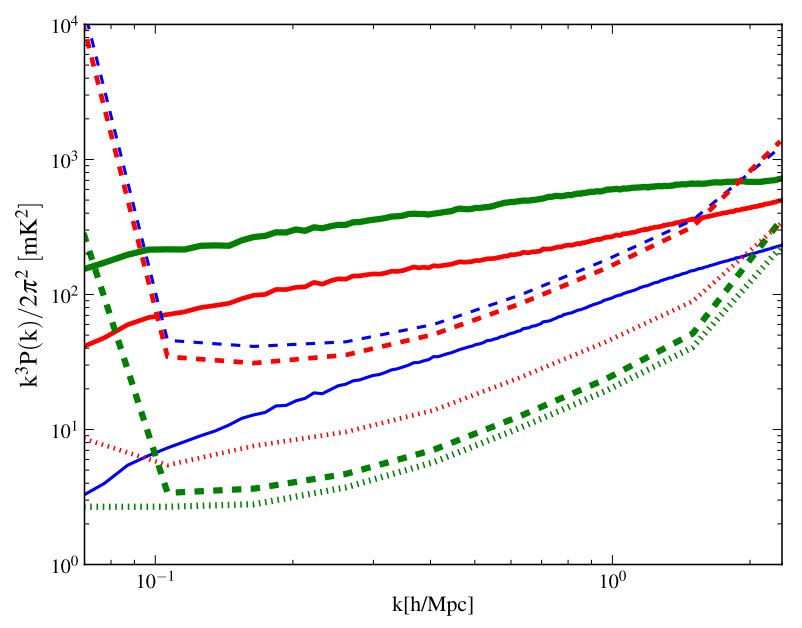

Fig. 13. Contributions for the $21 \mathrm{~cm}$ temperature power spectrum using simulations (solid lines), built using simulation $\mathrm{S} 1$ for $\left\langle x_{\alpha}\right\rangle=0.4$ $(z \sim 20.25)$. Dashed lines: expected errors. Dotted lines: expected errors assuming that $P_{\mu^{4}}$ is known. Solid lines from top to bottom are $P_{\mu^{0}}$ (green), $P_{\mu^{2}}$ (red) and $P_{\mu^{4}}$ (blue).

for by gridding the $\boldsymbol{k}$ space with a resolution along the line of sight set by the interval in frequency used for the analysis and a resolution perpendicular to the line of sight set by the field of view (see Sect. 3.2). The minimum size of the bins is given by this resolution. Note that the number of independent $\mu$ bins increase with $k$, although, as can be seen from Fig. 12, the errors will also increase due to the experimental noise. Moreover, the power spectrum is quite flat for small $k$ which will make it harder to extract the polynomial dependence (this is because the $P_{\mu^{0}}$ term is dominating in Eq. (15)).

Although difficult as we have seen, we can try to obtain $P_{\mu^{0}}, P_{\mu^{2}}$ and $P_{\mu^{4}}$ by fitting Eq. (15) to the measured $P(k, \mu)$ for $k \lesssim 1.0 \mathrm{~h} / \mathrm{Mpc}$. This in turn should allow us to obtain direct constraints on a combination of $P_{\delta_{x_{\alpha}} \delta_{x_{\alpha}}}$ and $P_{\delta \delta_{x_{\alpha}}}$. For small $k$ there are fewer bins so the uncertainty in $P_{\mu^{n}}$ will be high even for those scales where the errors in $P(k, \mu)$ are small. The expected errors on the $P_{\mu^{n}}$ term can be obtained using a Fisher matrix approach (Fisher 1935). For parameters $a, b=\left\{P_{\mu^{0}}, P_{\mu^{2}}, P_{\mu^{4}}\right\}$, the matrix is calculated through

$F_{a b}\left(k_{i}\right)=\sum_{j} \frac{1}{\Delta P\left(k_{i}, \mu_{j}\right)^{2}} \frac{\partial P\left(k_{i}, \mu_{j}\right)}{\partial a} \frac{\partial P\left(k_{i}, \mu_{j}\right)}{\partial b}$

where again $P(k, \mu)$ is given by Eq. (15) and $\Delta P\left(k_{i}, \mu_{j}\right)$ is the error for each bin. The covariance of the $P_{\mu^{n}}$ terms is just the inverse of the Fisher matrix and Fig. 13 shows the expected 1-sigma errors $\left(\sqrt{\left(F^{-1}\right)_{a a}}\right)$. We see that it should be possible to measure with reasonable accuracy both the $P_{\mu^{0}}$ and $P_{\mu^{2}}$ terms which can already give us interesting constraints on the Ly $\alpha$ field. In fact, if we fix the cosmology (e.g. $P_{\delta}$ ), then we should be able to extract information from $P_{\delta \delta_{x_{\alpha}}}$ and $P_{\delta_{x_{\alpha}} \delta_{x_{\alpha}}}$ separately. On the other hand, the $P_{\mu^{4}}$ will be hard to measure, which will make the extraction of cosmological information more difficult, simply because it is an order of magnitude smaller than the other terms on the scales we are focusing here.

In order to improve this last constraint we would have to increase the collecting area of the instrument and the frequency interval used (4 MHz in this case) so to have more modes along the line of sight (note however that this will lead to cosmological evolution of the signal along the frequency bin, as already discussed). The SKA should be able to measure modes up 
to $k \sim 10 \mathrm{~h} / \mathrm{Mpc}$ which could have higher values of $P_{\mu^{4}}$, however for $k>1 \mathrm{~h} / \mathrm{Mpc}$ the angular decomposition is no longer valid and the separate measurements of the $P(k, \mu)$ terms will be of little use (except maybe for foreground removal).

In this case, it might be better to just try to fit the parameters of the model directly to the averaged power spectrum $P(k)$ using the simulations (as shown in Fig. 8). If on the other hand we assume we already know $P_{\mu^{4}}$ with reasonable accuracy then the errors on $P_{\mu^{2}}$ will improve considerably (Fig. 13) and any degeneracies between $P_{\mu^{0}}$ and $P_{\mu^{2}}$ will be broken.

\subsection{Extracting the Poisson term}

Finally, by measuring the $P(k, \mu)$ terms, we can in principle use an estimator to probe the properties of the first sources of radiation directly through the Ly $\alpha$ field, without having to go through a full, model dependent, parameter fit to the data. This is based on the decomposition proposed in Barkana \& Loeb (2005a), valid in the linear regime. In that case, $\delta_{x_{\alpha}}$ can be expressed as a function of its dependence on effects correlated and uncorrelated with $\delta$ as:

$\delta_{x_{\alpha}}(\boldsymbol{k})=W(k) \delta(\boldsymbol{k})+\delta_{\mathrm{p}}(\boldsymbol{k})$.

Note that this decomposition is actually quite general and the main assumption here is the linearity on $\delta . \delta_{\mathrm{P}}$ is the Poisson contribution that arises from the statistical fluctuations in the number density of the rare first galaxies (galaxies being discrete objects and therefore not tracking the continuous density field perfectly) and which to first order is uncorrelated with the density fluctuations.

The window function, $W(k)$, contains several effects by which $\delta_{x_{\alpha}}$ is related to the underlying matter density fluctuations, $\delta$ (see Barkana \& Loeb 2005b for details). The strongest effect between $\delta$ and $\delta_{x_{\alpha}}$ is the biasing of the number density of galaxies with respect to the density fluctuations by a factor $b(z)$ (the average halo bias) so that an overdense region will have a factor $(1+b(z) \delta)$ more sources (Mo \& White 1996). This is the only term that is implicitly included in our simulations through the way we do the $3 \mathrm{~d}$ integration of the $\operatorname{Ly} \alpha$ flux. Note however that this term completely dominates over the other terms in Barkana \& Loeb (2005b) since $b(z) \sim 15$ at $z \sim 20$. In that case, we can express $W(k)$ as:

$W(k)=\frac{1}{\left\langle x_{\alpha}\right\rangle} \int_{z}^{z_{\max (2)}} \mathrm{d} z^{\prime} \frac{D\left(z^{\prime}\right)}{D(z)} \frac{\mathrm{d} x_{\alpha}}{\mathrm{d} z^{\prime}} b\left(z^{\prime}\right) \frac{\sin (k r)}{k r}$,

where $D(z)$ is the linear growth function and we are just considering photons emitted below the Lyman beta limit for comparison, so that $z_{\max (2)}=(32 / 27)(1+z)-1$ (Barkana \& Loeb 2005b; Pritchard \& Furlanetto 2007).

Using the parametrization of $\delta_{x_{\alpha}}$ we can write $P_{x_{\alpha}}(k)=$ $W^{2}(k) P_{\delta}(k)+P_{\mathrm{p}}(k)$ and $P_{\delta \delta_{x_{\alpha}}}(k)=W(k) P_{\delta}(k)$, meaning that we can isolate $W(k)$ from the simulations $\left(W(k)=P_{\delta \delta_{x_{\alpha}}}(k) / P_{\delta}(k)\right)$. In Fig. 14 we show the window function obtained from Eq. (22), with values from simulation S1, which has the same behavior as the one computed by Pritchard \& Furlanetto (2007). Figure 14 also shows that the window function obtained from Eq. (22) has a higher amplitude for large scales and small scales than the one obtained from simulation $\mathrm{S} 1$ using $P_{\delta}$ and $P_{\delta \delta_{x_{\alpha}}}$. On large scales this is probably a consequence of our box still not being quite large enough to encompass the rarest, most highly biased objects leading to an underestimate of the power on large scales. On small scales, this seems to be a consequence of the onset of non-linearities in the density and $\operatorname{Ly} \alpha$ fields. On scales close

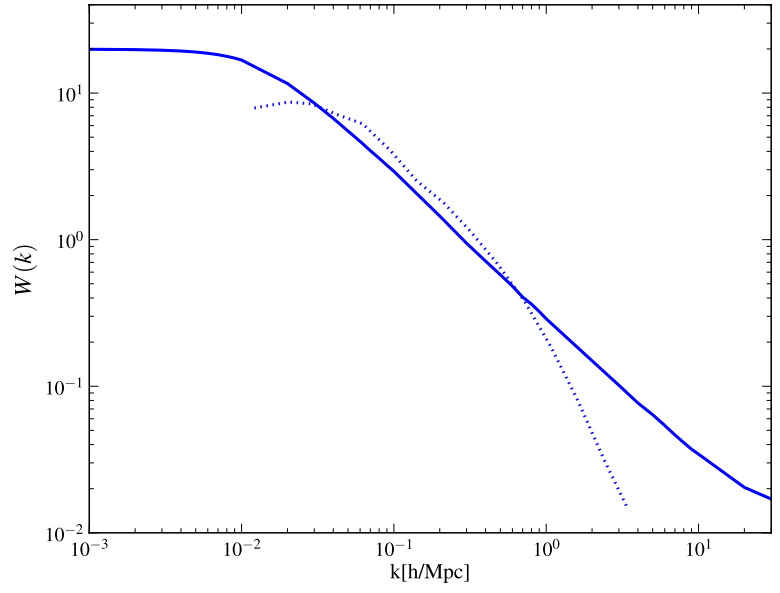

Fig. 14. Window function for simulation $\mathrm{S} 1$ considering only photons emitted below the Lyman beta limit, for $\left\langle x_{\alpha}\right\rangle=0.4(z \sim 20.25)$. Solid lines are obtained using Eq. (22) and dotted lines are obtained using $P_{\delta \delta_{x_{\alpha}}}(k)$ and $P_{\delta}(k)$.

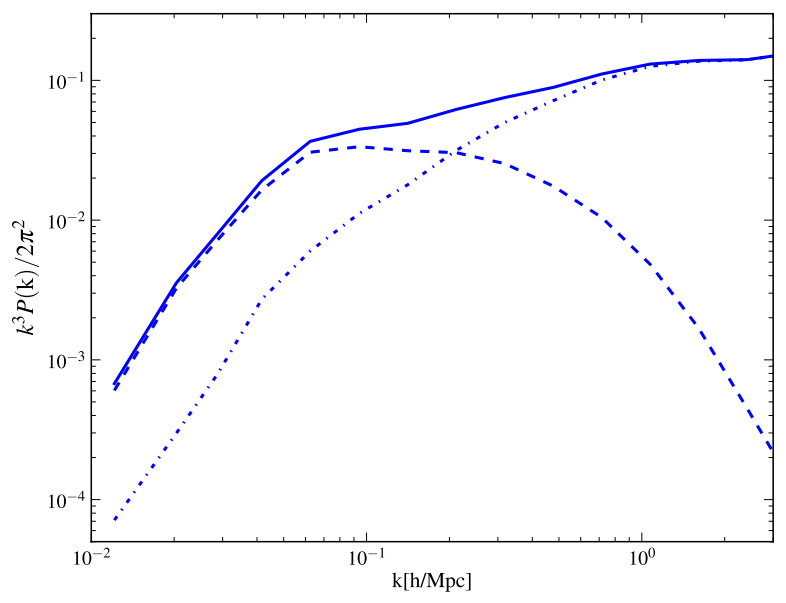

Fig. 15. Power spectra of fluctuations in $x_{\alpha}$ using simulation $\mathrm{S} 1$ for $\left\langle x_{\alpha}\right\rangle=0.4(z \sim 20.25)$. Shown are $P_{x_{\alpha}}$ (solid line), $W^{2} P_{\delta}$ (dashed line) and $P_{\mathrm{p}}($ dotted line $)$.

enough to clusters of sources that the Ly $\alpha$ coupling saturates, we might expect to see a steeper fall off in the $21 \mathrm{~cm}$ power as seen here. Nevertheless, $W(k)$ obtained from the simulation follows quite closely the expected theoretical values, which supports the decomposition given in Eq. (21) and validates the signal extraction we discuss next.

Also, in Fig. 15 we show $P_{\delta_{x_{\alpha}}}, W^{2} P_{\delta}$ and $P_{\mathrm{p}}$, built with $P_{\delta}$, $P_{\delta_{x_{\alpha}}}$ and $P_{\delta \delta_{x_{\alpha}}}$ from simulation S1. This shows the behavior expected from the analytic model of Barkana \& Loeb (2005b) and gives an amplitude of $P_{\mathrm{p}}$ that is broadly consistent with the values that we calculate from their model. The Poisson contribution dominates for $k>0.2 \mathrm{~h} / \mathrm{Mpc}$ so that there is just a small interval where we can expect to probe this term before non-linear effects correlated to the density field become relevant. Note that the Poisson contribution flattens on small scales $k \gtrsim 1$ (h/Mpc) slightly more than predicted by the analytic model. This is similar to the drop off in power on small scales seen in $W(k)$ and probably occurs for similar reasons.

For large enough scales, we can use the observational data to obtain the window function through

$\frac{P_{\mu^{2}}(k)}{P_{\mu^{4}}(k)}=2 \times\left[1+\beta+\frac{1}{1+\left\langle x_{\alpha}\right\rangle} W(k)\right]$, 


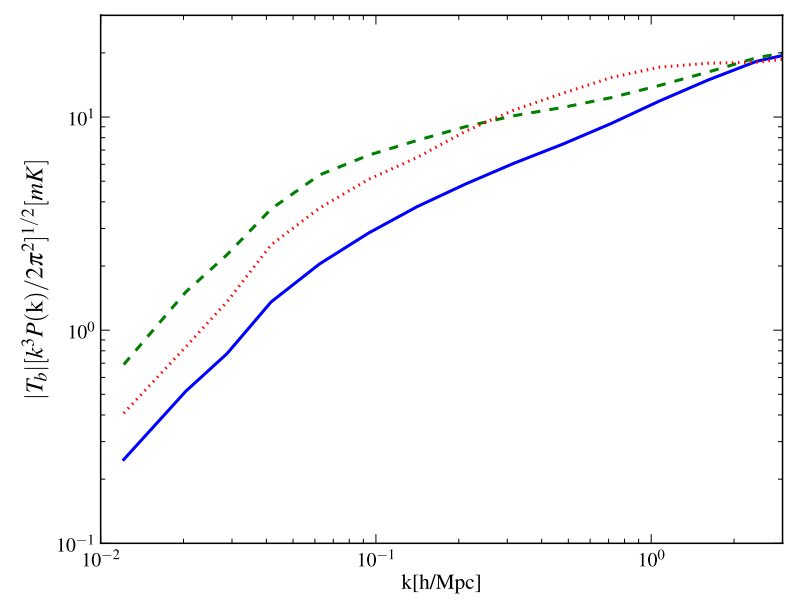

Fig. 16. Power spectra of brightness temperature fluctuations using simulation S1 for $\left\langle x_{\alpha}\right\rangle=0.4(z \sim 20.25)$. Shown are $P_{\mu^{2}}$ (green dashed line) $2 C(z) P_{\delta}$ (blue solid line), and $P_{u n-\delta}$ (red dotted line).

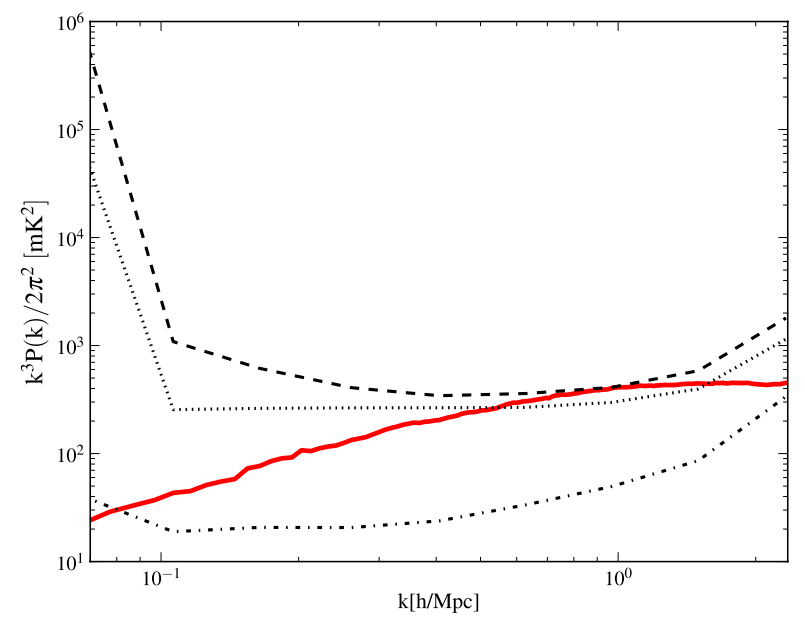

Fig. 17. Power spectra of $C^{2}(z)\left(1 /\left(1+\left\langle x_{\alpha}\right\rangle\right)\right)^{2} P_{\mathrm{p}}(k)$ (solid line), built using simulation $\mathrm{S} 1$ for $\left\langle x_{\alpha}\right\rangle=0.4(z \sim 20.25)$. Expected error (dashed line) and expected error assuming that $P_{\delta}$ is known (dotted line), so the error in $P_{\mu^{4}}$ comes only from $C(z)$. Dot-dashed line assumes that $C(z)$ is known to high accuracy.

and the Poisson contribution uncorrelated with $\delta$ :

$P_{u n-\delta}(k)=P_{\mu^{0}}(k)-\frac{P_{\mu^{2}}^{2}(k)}{4 P_{\mu^{4}}(k)}=C^{2}(z)\left(\frac{1}{1+\left\langle x_{\alpha}\right\rangle}\right)^{2} P_{\mathrm{p}}(k)$.

The use of Eqs. (23) and (24) allows us to characterize the Ly $\alpha$ background and the number and distribution of galaxies at these early redshifts. In Fig. 16, we used simulation S1 to show $P_{\mathrm{un}-\delta}$ and $P_{\mu^{2}}$, which are consistent with the results obtained by Barkana \& Loeb (2005b). Figure 17 shows the power spectra of the Poisson contribution (Eq. (24)) and the expected errors associated with this measurement. As expected, due to the high error in $P_{\mu^{4}}$, the measurement of this Poisson term will be quite difficult even with the assumed experimental setup (dashed line). If we assume that the matter power spectrum is known with reasonable accuracy from $\mathrm{CMB}$ data and galaxy surveys, then we can combine the measurement of the $P_{\mu^{4}}$ term at several $k$ to constrain $C^{2}(z)$ (Eq. (16)), e.g. the mean brightness temperature on the sky. Unfortunately the constraint is still large,
$C^{2}(z) \approx(6.42 \pm 5.60) \times 10^{3} \mathrm{mK}^{2}$, which translates into large errors for the Poisson term (dotted lines). Nevertheless, a detection will still be possible, with a signal to noise of 2.6 (dashed line) and 3.8 (dotted line).

If we further assume that we combine this result with the measurements from a global experiment in order to measure the mean brightness temperature with high accuracy, then we can extract all the relevant power spectra reasonably well (dotdashed line).

\section{Conclusions}

In this paper, we have made use of a recently developed fast semi-numerical code to explore the possibility for $21 \mathrm{~cm}$ observations of the time of the first galaxies with SKA. We demonstrated that this code allows the simulation of the full range of scales likely to be accessible to observations. Here we have focused on the impact of $21 \mathrm{~cm}$ fluctuations from large fluctuations in the $\operatorname{Ly} \alpha$ coupling, which arise due to the clustering of the rare first galaxies and considered different emission models.

Measurement of these fluctuations would provide insight into the formation of the very first galaxies and would be highly complementary with the next generation of large optical/IR telescopes. We have shown that SKA-pathfinders with $\sim 10 \%$ of the full collecting area should be capable of making a statistical detection of the $21 \mathrm{~cm}$ power spectrum at redshifts $z \lesssim 20$. With the full SKA sensitivity this detection would become a measurement allowing astrophysical properties of the first galaxies to be determined. Note that it is crucial that these experiments are able to go below $70 \mathrm{MHz}$ in order to probe the signal.

Observations with an SKA-like instrument would enable the determination of $\left\langle x_{\alpha}\right\rangle$ as a function of redshift from the amplitude and shape of the $21 \mathrm{~cm}$ fluctuations. Although different models with the same $\left\langle x_{\alpha}\right\rangle$ give similar power spectra, this evolution with redshift should allow to distinguish them. Even though there is a strong degeneracy between the star formation rate and the UV spectral properties of the galaxies themselves, these observations would enable constraints to be placed on the star formation rate in the earliest generations of galaxies. Although crude, the resulting "Madau plot" would be very useful for understanding the formation processes of the first galaxies.

Moving beyond the angle-averaged power spectrum, we have investigated the use of redshift-space distortions to separate out different components of the power spectrum as suggested by Barkana \& Loeb (2005b). These measurements are difficult due to instrumental limitations and are further complicated by non-linearities on small scales. For the first time, we have investigated the non-linearity of $\operatorname{Ly} \alpha$ fluctuations showing that they should be important on scales $k \gtrsim 0.2(\mathrm{~h} / \mathrm{Mpc}$ ). Nonetheless, on larger scales the detection of these effects is possible with SKA level sensitivity and should allow to put direct constraints on the Ly $\alpha$ power spectrum.

Redshift space distortions further offer the possibility of extracting components of the power spectrum that do not correlate with the density field. This Poissonian component contains direct information about the number density of sources and so gives complementary information to the other sources of fluctuations. We have shown that a detection is possible and if strong assumptions about the underlying cosmology are possible and combined with information about the mean $21 \mathrm{~cm}$ signal, then these fluctuations may be picked out clearly by SKA.

These results illustrate the potential of $21 \mathrm{~cm}$ observations to shed new light on the astrophysics during the pre-Reionization epoch. In the future, our improved knowledge of cosmological 
parameters will provide a firm foundation to pick out the details of galaxy formation in the early Universe. SKA and pathfinders capable of observing at frequencies $v \lessgtr 100 \mathrm{MHz}$ will begin to access this interesting period and transform our understanding of the cosmic dawn.

Acknowledgements. This work was partially supported by FCT-Portugal under grants PTDC/FIS/66825/2006 and PTDC/FIS/100170/2008. J.R.P. is supported by NASA through Hubble Fellowship grant HST-HF-01211.01-A awarded by the Space Telescope Science Institute, which is operated by the Association of Universities for Research in Astronomy, Inc., for NASA, under contract NAS 5-26555. A.C. acknowledges support of NSF grant CAREER AST-0645427. M.G.S. was a visitor at UCI when this work was concluded.

\section{References}

Baek, S., Di Matteo, P., Semelin, B., Combes, F., \& Revaz, Y. 2009, A\&A, 495, 389

Baek, S., Semelin, B., Di Matteo, P., Revaz, Y., \& Combes, F. 2010, 523, A4

Barkana, R., \& Loeb, A. 2001, Phys. Rep., 349, 125

Barkana, R., \& Loeb, A. 2004, ApJ, 609, 474

Barkana, R., \& Loeb, A. 2005a, ApJ, 624, L65

Barkana, R., \& Loeb, A. 2005b, ApJ, 626, 1

Bowman, J. D., Morales, M. F., \& Hewitt, J. N. 2006, ApJ, 638, 20

Bowman, J. D., Morales, M. F., \& Hewitt, J. N. 2007, ApJ, 661, 1

Bromm, V., \& Larson, R. B. 2004, ARA\&A, 42, 79

Chuzhoy, L., \& Zheng, Z. 2007, ApJ, 670, 912

Cohen, A. S., \& Röttgering, H. J. A. 2009, AJ, 138, 439

Cohn, J. D., \& White, M. 2008, MNRAS, 385, 2025

Cuby, J., Hibon, P., Lidman, C., et al. 2007, A\&A, 461, 911

Faulkner, A. J. E. A. 2010, SKADS Memos: http://www.skads-eu.org Field, G. B. 1959, ApJ, 129, 536

Fisher, R. 1935, J. Roy. Stat. Soc., 98, 39

Furlanetto, S. R., Oh, S. P., \& Briggs, F. H. 2006, Phys. Rep., 433, 181

Garrett, M. A., Cordes, J. M., Deboer, D. R., et al. 2010 [arXiv: 1008. 2871]

Harker, G., Zaroubi, S., Bernardi, G., et al. 2010, MNRAS, 405, 2492

Heitmann, K., Lukic, Z., Fasel, P., et al. 2008, Comp. Science and discov., 1, 015003

Hibon, P., Cuby, J., Willis, J., et al. 2010, A\&A, 515, A97

Hirata, C. M. 2006, MNRAS, 367, 259

Jelić, V., Zaroubi, S., Labropoulos, P., et al. 2008, MNRAS, 389, 1319

Kaiser, N. 1987, MNRAS, 227, 1

Komatsu, E., Smith, K. M., Dunkley, J., et al. 2011, ApJS, 192, 18
Leitherer, C., Schaerer, D., Goldader, J. D., et al. 1999, ApJS, 123, 3

Lewis, A., Challinor, A., \& Lasenby, A. 2000, ApJ, 538, 473

Lidz, A., Zahn, O., McQuinn, M., Zaldarriaga, M., \& Hernquist, L. 2008, ApJ, 680,962

Liu, A., Tegmark, M., Morrison, S., Lutomirski, A., \& Zaldarriaga, M. 2010, MNRAS, 408, 1029

Lukic, Z., Heitmann, K., Habib, S., Bashinsky, S., \& Ricker, P. M. 2007, ApJ, 671,1160

Madau, P., Ferguson, H. C., Dickinson, M. E., et al. 1996, MNRAS, 283, 1388

Mao, Y., Tegmark, M., McQuinn, M., Zaldarriaga, M., \& Zahn, O. 2008, Phys. Rev. D, 78, 023529

Matejek, M. S., \& Morales, M. F. 2009, ArXiv e-prints

McMahon, R., Parry, I., Venemans, B., et al. 2008, The Messenger, 131, 11

McQuinn, M., Zahn, O., Zaldarriaga, M., Hernquist, L., \& Furlanetto, S. R. 2006, ApJ, 653, 815

Mesinger, A., Furlanetto, S., \& Cen, R. 2010 [arXiv: 1003 . 3878]

Mo, H. J., \& White, S. D. M. 1996, MNRAS, 282, 347

Morales, M. F., Bowman, J. D., \& Hewitt, J. N. 2006, ApJ, 648, 767

Nilsson, K. K., Orsi, A., Lacey, C. G., Baugh, C. M., \& Thommes, E. 2007, A\&A, 474, 385

Ouchi, M., Shimasaku, K., Akiyama, M., et al. 2008, ApJS, 176, 301

Pritchard, J. R., \& Furlanetto, S. R. 2006, MNRAS, 367, 1057

Pritchard, J. R., \& Furlanetto, S. R. 2007, MNRAS, 376, 1680

Pritchard, J. R., \& Loeb, A. 2008, Phys. Rev. D, 78, 103511

Pritchard, J. R., \& Loeb, A. 2010, Phys. Rev. D, 82, 023006

Reed, D. S., Bower, R., Frenk, C. S., Jenkins, A., \& Theuns, T. 2007, MNRAS, 374, 2

Salvaterra, R., Ferrara, A., \& Dayal, P. 2010 [arXiv: 1003. 3873]

Santos, M. G., Cooray, A., \& Knox, L. 2005, ApJ, 625, 575

Santos, M. G., Amblard, A., Pritchard, J., et al. 2008, ApJ, 689, 1

Santos, M. G., Ferramacho, L., Silva, M. B., Amblard, A., \& Cooray, A. 2010, MNRAS, 406, 2421

Schaerer, D. 2003a, A\&A, 397, 527

Schaerer, D. 2003b, A\&A, 397, 527

Schilizzi, R. T., Alexander, P., Cordes, J. M., et al. 2007, SKA documents: http: //www. skatelescope.org

Semelin, B., Combes, F., \& Baek, S. 2007, A\&A, 474, 365

Shaw, J. R., \& Lewis, A. 2008, Phys. Rev. D, 78, 103512

Stark, D. P., Ellis, R. S., Richard, J., et al. 2007, ApJ, 663, 10

Stiavelli, M., Fall, S. M., \& Panagia, N. 2004, ApJ, 610, L1

Trac, H., \& Cen, R. 2007, ApJ, 671, 1

Trac, H., \& Pen, U.-L. 2004, New Astron., 9, 443

Trac, H., \& Pen, U.-L. 2006, New Astron., 11, 273

Trac, H., Cen, R., \& Loeb, A. 2008, ApJ, 689, L81

Willis, J. P., Courbin, F., Kneib, J., \& Minniti, D. 2008, MNRAS, 384, 1039

Wouthuysen, S. A. 1952, AJ, 57, 31

Zahn, O., Lidz, A., McQuinn, M., et al. 2007, ApJ, 654, 12 